\title{
Molecular hydrogen in the circumstellar environments of Herbig Ae/Be stars probed by FUSE`
}

\author{
C. Martin-Zaïdi ${ }^{1}$, M. Deleuil ${ }^{2}$, J. Le Bourlot ${ }^{3}$, J.-C. Bouret ${ }^{2}$, A. Roberge ${ }^{4}$, C. P. Dullemond ${ }^{5}$, L. Testi ${ }^{6}$, P. D. Feldman ${ }^{7}$, \\ A. Lecavelier des Etangs ${ }^{8}$, and A. Vidal-Madjar ${ }^{8}$
}

1 Laboratoire d'Astrophysique de Grenoble, CNRS, Université Joseph-Fourier, UMR 5571, Grenoble, France e-mail: claire.martin-zaidi@obs.ujf-grenoble.fr

2 Laboratoire d'Astrophysique de Marseille, BP 8, Les trois Lucs, 13376 Marseille Cedex 12, France

3 LUTH, Observatoire de Paris, Université Paris 7, 92195 Meudon Cedex, France

${ }^{4}$ NASA Goddard Space Flight Center, Greenbelt, MD 20771, USA

5 Max-Planck-Institut für Astronomie, Königstuhl 17, 69117 Heidelberg, Germany

6 Osservatorio Astrofisico di Arcetri, Largo E. Fermi 5, 50125 Firenze, Italy

7 Department of Physics and Astronomy, JHU, Baltimore, MD 21218, USA

8 Institut d'Astrophysique de Paris, CNRS, 98bis boulevard Arago, 75014 Paris, France

Received 24 December 2007 / Accepted 13 March 2008

\begin{abstract}
Context. Molecular hydrogen $\left(\mathrm{H}_{2}\right)$ gas is the most abundant molecule in the circumstellar (CS) environments of young stars. It is thus a key element in our understanding of the evolution of pre-main sequence stars and their environments towards the main sequence. Aims. At the present time, little is known about the gas as compared to the dust in the environments of young stars. We thus observed molecular hydrogen around a sample of pre-main sequence stars in order to better characterize their gaseous CS environments.

Methods. The FUSE (Far Ultraviolet Spectroscopic Explorer) spectral range offers access to electronic transitions of $\mathrm{H}_{2}$. We analyzed the FUSE spectra of a sample of Herbig Ae/Be stars (HAeBes) covering a broad spectral range (from F4 to B2), including the mainsequence A5 star $\beta$ Pictoris. To better diagnose the origin of the detected molecular gas and its excitation conditions, we used a model of a photodissociation region.

Results. Our analysis demonstrates that the excitation of $\mathrm{H}_{2}$ is clearly different around most of the HAeBes compared to the interstellar medium. Moreover, the characteristics of $\mathrm{H}_{2}$ around Herbig Ae and Be stars give evidence for different excitation mechanisms. For the most massive stars of our sample (B8 to B2 type), the excitation diagrams are reproduced well by a model of photodissociation regions (PDR). Our results favor an interpretation in terms of large CS envelopes, remnants of the molecular clouds in which the stars were formed. On the other hand, the group of Ae stars (later than B9 type) known to possess disks is more inhomogeneous. In most cases, when $\mathrm{CS} \mathrm{H}_{2}$ is detected, the lines of sight do not pass through the disks. The excitation conditions of $\mathrm{H}_{2}$ around Ae stars cannot be reproduced by PDR models and correspond to warm and/or hot excited media very close to the stars. In addition, no clear correlation has been found between the ages of the stars and the amount of circumstellar $\mathrm{H}_{2}$. Our results suggest structural differences between Herbig Ae and Be star environments. Herbig Be stars do evolve faster than Ae stars, and consequently, most Herbig Be stars are younger than Ae ones at the time we observe them. It is thus more likely to find remnants of their parent cloud around them.
\end{abstract}

Key words. stars: circumstellar matter - stars: formation - stars: pre-main sequence - ISM: molecules

\section{Introduction}

A longstanding problem in modern astrophysics is how stars and planets form from their parent molecular clouds. It is generally accepted that the collapse of an interstellar molecular cloud core to form a protostar naturally produces a disk-shaped nebula in which planets might form. In the past twenty years, both planets and disks have been observed around nearby stars. Some young stars with disks, e.g., the T Tauri star GM Aur (Rice et al. 2003), are suspected of harboring young planets, and recently a massive planet orbiting TW Hya, a young star surrounded by a wellstudied disk, has been discovered by means radial velocity techniques (Setiawan et al. 2008).

Disks have been directly imaged around T Tauri stars (e.g., HK Tau, Stapelfeldt et al. 1998), while direct or coronagraphic

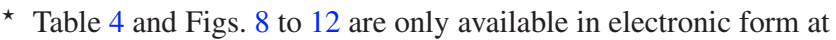
http://www . aanda.org imaging of Herbig Ae/Be star disks has been performed successfully only in a few cases (e.g. Pantin et al. 2000; Grady et al. 2001; Augereau \& Papaloizou 2004; Lagage et al. 2006). Most evidence of disks around Herbig Ae/Be stars is indirect: blueshifted absorption lines, polarization, mass estimates from millimeter observations, extinction measurements, and simple disk-model fitting to infrared spectral energy distributions (IR SEDs) (Bastien \& Menard 1990; Hillenbrand et al. 1992; Corcoran \& Ray 1997). Numerous observations have revealed elongated structures around several HAeBes, with velocity gradients along the major axes characteristic of gas in Keplerian rotation (Mannings \& Sargent 1997, 2000). In addition, the SEDs of some HAeBes show dips near $10 \mu \mathrm{m}$ that cannot be explained by normal dust-removal processes, such as the Poynting-Robertson drag and radiation pressure from the star. It instead suggests that the dust structure is evolving because of the disk breaking up. One possibility is that this is caused by planet formation (Bouwman et al. 2003). In this context, HAeBes are 
possible precursors of $\beta$ Pictoris and Vega-type stars, whose CS debris disks are believed to host planetary bodies. This raises the possibility that the environment around the HAeBes truly represents a very early phase of planet formation. The physical parameters of HAeBes disks can thus be used to constrain the duration of the protoplanetary phase and the age at which the signatures of planets become visible in CS disks.

On the other hand, mid-IR observations at high spatial resolution have revealed that the emission observed from HAeBes is generally not confined to optically thick disks but instead comes from more complex environments such as remnant envelopes (Polomski et al. 2002). On the basis of millimeter interferometric measurements, Natta et al. (2000) conclude that CS envelopes are much more common around Herbig Be stars (HBes) than around Herbig Ae/B9 stars (HAes). These conclusions have been confirmed by near-IR speckel interferometry (Leinert et al. 2001). This indicates structural differences between Herbig Ae stars and Herbig Be stars. These results are fully compatible with the faster evolution of the more massive HBes. Indeed, due to their stronger radiation fields, the CS environments around HBes evolve faster than around HAes, which translates especially into a rapid depletion of the CS disk material. Consequently, the average $\mathrm{HBe}$ stars are younger than HAes at the time we observe them; therefore, it is more likely to find larger amounts of remnant CS material around Herbig Be stars, since less time is available to dissipate it. In this scenario, it is expected that the CS material is mostly concentrated at the outer edges of the close CS environment and distributed in more or less spherically envelopes.

Molecular hydrogen, from which giant planets are primarily believed to form, is the most abundant molecule in the CS environments of young stars. The detection of $\mathrm{H}_{2}$ provides the most direct information about the gaseous content of the CS environments of HAeBes, setting limits on the timescales for the dissipation of CS matter and possible planet building. Tentative detections of pure rotational $\mathrm{H}_{2}$ lines at infrared wavelengths towards a few Herbig stars and T Tauri stars have been previously obtained with ISO-SWS (Thi et al. 2001). But groundbased observations have shown that contamination from surrounding cloud material can be important and that ISO could not have detected disk gas (Richter et al. 2002; Sako et al. 2005). The $\mathrm{H}_{2}$ mid-IR emission lines have been clearly detected in the disks of only two Herbig stars, namely AB Aur (Bitner et al. 2007) and HD 97048 (Martin-Zaïdi et al. 2007). These studies show that the gas has not dissipated in the inner parts of these disks with ages of about $3 \mathrm{Myr}$ and that peculiar $\mathrm{H}_{2}$ excitation conditions are present in the disks. The electronic transitions of $\mathrm{H}_{2}$, which are much stronger than the rotational ones, occur in the far-ultraviolet (FUV) spectral range, a domain covered by FUSE (Far Ultraviolet Spectrocopic Explorer). Previous FUSE observations of selected young stars harboring CS disks (Lecavelier des Etangs et al. 2001, 2003; Roberge et al. 2001) have demonstrated that such a study can make significant contributions clarifying issues raised in previous work.

We present the results of our systematic analysis of the FUSE spectra of a large group of Herbig HAeBe stars with a variety of spectral types and Vega-type stars, including $\beta$ Pictoris. This paper is organized as follows. In Sect. 2 we present our sample stars and recall the methods used for data reduction and analysis, which have been presented in previous papers. The results obtained from the $\mathrm{H}_{2}$ analysis are given in Sect. 3. The detailed analysis and interpretation of our results for each group of stars are presented in Sects. 4 and 5. We explore possible evolutionary trends in our results in Sect. 6. Our conclusions are discussed in Sect. 7.

\section{The sample stars}

To perform this study, we have compiled a set of observations from different programs we carried out over the past six years, supplemented by some data from the archives (Table 1). This results in a sample of 18 HAeBes with spectral types from F4 to B2 and one main-sequence star, $\beta$ Pictoris, prototype of the Vega-type stars. Two stars, HD 141569A and HD 109573, belong to the so-called transitional class and are passing from the pre-main sequence Herbig star stage to the zero-age main sequence (ZAMS) stars. Both are known to possess CS disks (Jura 1991; Augereau et al. 1999) with ages close to about $10 \mathrm{Myr}$ (Table 2).

Our sample also includes HD 135344. The spectral type of this star has not been determined precisely in the literature, estimates ranging from $\mathrm{F} 4$ to $\mathrm{A} 0$, but it has been classified as a Herbig star (e.g. Thé et al. 1994; Malfait et al. 1998; Thi et al. 2001). In the rest of this paper, we thus include this star in the sub sample called Herbig Ae/B9 stars.

For several stars, some early results of the FUSE spectral analysis have already been published, and we will reiterate the results obtained from these previous studies. The FUSE observations of $11 \mathrm{HAeBes}$ are presented here for the first time. Some stars have been observed twice (e.g. AB Aur) or even three times (e.g. $\beta$ Pictoris, HD 104237) with a time separation between the FUSE observations ranging from about one month to more than one year. For the sake of consistency, we thus re-did the spectral analysis of the entire sample and took advantage of any new observations to improve the $S / N$ of the spectra and the $\mathrm{H}_{2}$ spectral analysis.

The fundamental parameters of the stars are given in Table 2. Most are from the literature, but in some cases, we re-evaluated the effective temperatures of the stars (for details on the method, see Bouret et al. 2003). Using these values and the Palla \& Stahler (1993) pre-main sequence tracks, we calculated the ages and masses using interpolation routines written by Testi et al. (1998). All the stars are plotted on the HR diagram presented in Fig. 1, showing their evolutionary status.

All the stars were observed with the FUSE $30^{\prime \prime} \times 30^{\prime \prime}$ LWRS aperture at a resolution of about 15000 . The FUSE observations cover the wavelength range from $905 \AA$ to $1187 \AA$. For the sake of consistency, all the spectra were reprocessed with version 3.0.7 of the FUSE calibration pipeline, CalFUSE (Dixon et al. 2007). For each star, the total exposure time was split into several sub-exposures which have been co-aligned using a linear cross-correlation procedure and added segment by segment. In some cases, the co-added spectra in each detector channel were rebinned in wavelength to increase the $S / N$ ratio without degrading the resolution.

\section{Molecular hydrogen analysis}

\section{1. $\mathrm{H}_{2}$ spectral analysis}

The FUSE spectral domain offers access to the Lyman and Werner series electronic transitions of $\mathrm{H}_{2}$. These electronic transitions are between the rotational levels $(J)$ of the ground vibrational level $(v=0)$ of the fundamental electronic level $(X)$ and the rotational levels of the vibrational levels of the first $(B)$ and second $(C)$ excited electronic levels. Under certain conditions, spectral lines corresponding to transitions from the rotational 
Table 1. Log of the observations.

\begin{tabular}{|c|c|c|c|c|c|c|c|}
\hline Star & $\begin{array}{c}\alpha \\
(2000)\end{array}$ & $\begin{array}{c}\delta \\
(2000)\end{array}$ & $\begin{array}{c}F U S E \\
\text { program }\end{array}$ & $\begin{array}{l}\text { Principal } \\
\text { investigator }{ }^{(a)}\end{array}$ & $\begin{array}{c}\text { Total observing } \\
\text { time }(\mathrm{ks})\end{array}$ & $\begin{array}{c}F U S E \\
\text { aperture }\end{array}$ & $\begin{array}{c}\begin{array}{c}\text { Published } \\
\text { data }\end{array}(b) \\
\end{array}$ \\
\hline \multirow[t]{3}{*}{$\beta$ Pictoris } & 054717.09 & -510359.45 & P219 & A. Vidal-Madjar & 24.78 & LWRS & $(1)$ \\
\hline & & & Q119 & M. Deleuil & 34.50 & LWRS & \\
\hline & & & $\mathrm{C} 132$ & J.-C. Bouret & 47.79 & LWRS & \\
\hline HD 135344 & 151548.44 & -370916.03 & Q306 & A. Lecavelier des Etangs & 8.44 & LWRS & \\
\hline HD 100453 & 113305.57 & -541928.54 & $\mathrm{C} 126$ & archives & 11.79 & LWRS & \\
\hline HD 36112 & 053027.53 & +251957.08 & Q319 & M. Deleuil & 7.10 & LWRS & \\
\hline \multirow[t]{2}{*}{ HD 104237} & 120005.08 & -781134.56 & $\mathrm{P} 163$ & archives & 18.97 & LWRS & \\
\hline & & & P263 & archives & 20.84 & LWRS & \\
\hline \multirow[t]{2}{*}{ HD 163296} & 175621.29 & -215721.88 & P219 & A. Vidal-Madjar & 15.92 & LWRS & (2) \\
\hline & & & Q219 & A. Lecavelier des Etangs & 16.16 & LWRS & \\
\hline NX PUP & 071928.26 & -443511.28 & Z906 & Observatory Program & 17.80 & LWRS & \\
\hline \multirow[t]{2}{*}{ AB Aur } & 045545.84 & +303304.29 & P119 & A. Vidal-Madjar & 13.66 & LWRS & (3) \\
\hline & & & P219 & A. Vidal-Madjar & 15.55 & LWRS & \\
\hline HD 141569 & 154957.75 & -035516.36 & Q319 & M. Deleuil & 6.79 & LWRS & (4) \\
\hline \multirow[t]{2}{*}{ HD 100546} & 113325.44 & -701141.24 & P119 & A. Vidal-Madjar & 10.35 & LWRS & (5) \\
\hline & & & P219 & A. Vidal-Madjar & 11.94 & LWRS & \\
\hline HD 109573 & 123601.03 & -395210.22 & B091 & archives & 12.38 & LWRS & \\
\hline \multirow[t]{2}{*}{ HD 176386} & 190138.93 & -365326.55 & P119 & A. Vidal-Madjar & 14.98 & LWRS & \\
\hline & & & P219 & A. Vidal-Madjar & 27.39 & LWRS & \\
\hline HD 250550 & 060159.99 & +163056.73 & B038 & C. Catala & 9.06 & LWRS & (6) \\
\hline HD 85567 & 095028.54 & -605802.96 & Z906 & Observatory Program & 7.06 & LWRS & \\
\hline HD 259431 & 063305.19 & +101919.98 & B038 & C. Catala & 16.68 & LWRS & (6) \\
\hline \multirow[t]{2}{*}{ HD 38087} & 054300.57 & -021845.37 & A063 & archives & 31.12 & LWRS & \\
\hline & & & P116 & archives & 4.00 & MDRS & \\
\hline HD 76534 & 085508.71 & -432759.86 & B038 & C. Catala & 5.71 & LWRS & (7) \\
\hline
\end{tabular}

${ }^{(a)}$ Most of the data were obtained by our Team (through guaranteed time and guest investigator observations). Some of the data are from the FUSE archives or from observatory programs dedicated to instrument testing.

(b) Data already published.

References: (1) Lecavelier des Etangs et al. (2001); (2) Deleuil et al. (2005); (3) Roberge et al. (2001); (4) Martin-Zaïdi et al. (2005); (5) Deleuil et al. (2004); (6) Bouret et al. (2003); (7) Martin et al. (2004).

Table 2. Physical parameters of the sample stars.

\begin{tabular}{|c|c|c|c|c|c|c|c|c|c|c|}
\hline Name & $\begin{array}{c}\text { Spectral } \\
\text { type }\end{array}$ & $\begin{array}{l}T_{\text {eff }} \\
(\mathrm{K})\end{array}$ & $E(B-V)$ & $\begin{array}{c}v \sin i \\
\left(\mathrm{~km} \mathrm{~s}^{-1}\right)\end{array}$ & $\begin{array}{r}v_{\text {rad }} \\
\left(\mathrm{km} \mathrm{s}^{-1}\right) \\
\end{array}$ & $\begin{array}{r}\text { Age }^{(b)} \\
(\mathrm{Myr})\end{array}$ & $\begin{array}{c}\text { Mass }^{(b)} \\
\left(M_{\odot}\right)\end{array}$ & $\begin{array}{r}\text { Distance } \\
(\mathrm{pc}) \\
\end{array}$ & $\begin{array}{c}\log L_{*} \\
\left(L_{\odot}\right)\end{array}$ & Ref. \\
\hline$\beta$ Pictoris & $\mathrm{A} 5 \mathrm{~V}$ & 8200 & 0.02 & 140 & +20 & 20 & 1.75 & 19.3 & 0.94 & $1,2,3,4,5$ \\
\hline HD 135344 & F4V & 6750 & 0.15 & 80 & -3 & 30 & 1.3 & 84 & 0.48 & $1,6,7,8,9,11$ \\
\hline HD 100453 & A9 & 7500 & 0.08 & 39 & - & 10 & 1.7 & 114 & 0.95 & $1,7,8,10,11$ \\
\hline HD 36112 & A5 & 8120 & 0.07 & 60 & +17.6 & 5.0 & 2.0 & 200 & 1.35 & $1,9,11,12,13$ \\
\hline HD 104237 & A4 & 8500 & 0.10 & 10 & +14 & 2.4 & 2.4 & 116 & 1.55 & $1,7,11,12,14$ \\
\hline HD 163296 & A1Ve & 8700 & 0.06 & 133 & +4 & 4.7 & 2.2 & 122 & 1.48 & $1,12,15,16$ \\
\hline NX PUP & $\mathrm{A} 0$ & 9770 & 0.19 & 120 & - & 2.8 & 2.4 & 450 & 1.63 & $1,12,18,19,20$ \\
\hline AB Aur & A0V & 9800 & 0.16 & 100 & +21 & 2.6 & 2.4 & 144 & 1.68 & $1,23,24,25$ \\
\hline HD 141569 & B9V & 10040 & 0.13 & 236 & -6.4 & $\geq 5.0$ & 2.3 & 108 & 1.35 & $1,12,28$ \\
\hline HD 100546 & B9V & 10470 & 0.08 & 55 & +17 & $\geq 9.0$ & 2.4 & 103 & 1.51 & $1,11,12,29$ \\
\hline HD 109573 & A0/B9 & 10500 & 0.03 & 152 & +9.4 & $\geq 8.0$ & 2.2 & 67 & 1.37 & $1,17,21,22$ \\
\hline HD 176386 & B9/B8 & $12000^{(a)}$ & 0.19 & 220 & +7.3 & 3.5 & 2.7 & 140 & 1.69 & 1,30 \\
\hline HD 250550 & B7 & $12800^{(a)}$ & 0.22 & $110^{(a)}$ & +31 & 1.0 & 3.6 & $\geq 160$ & 2.19 & $1,12,23,32$ \\
\hline HD 85567 & B5V & $15200^{(a)}$ & 0.23 & $60^{(a)}$ & $0 /-5$ & 1.0 & 4.2 & $\geq 480$ & 2.54 & $1,12,33$ \\
\hline HD 259431 & B5 & $15900^{(a)}$ & 0.26 & $95^{(a)}$ & +43 & $\geq 1.0$ & 4.4 & $290-800$ & 2.52 & $1,12,23,32$ \\
\hline HD 38087 & B5V & $16500^{(a)}$ & 0.29 & $100^{(a)}$ & +33 & $\geq 1.0$ & 4.6 & 381 & 2.61 & $1,14^{(c)}$ \\
\hline HD 76534 & B2 & $20000^{(a)}$ & 0.32 & $110^{(a)}$ & +17 & $\geq 0.5$ & $\geq 5$ & $\geq 160$ & 2.83 & $1,12,23,34$ \\
\hline
\end{tabular}

(a) $T_{\text {eff }}$ and $v \sin i$ determined from our modeling (see Bouret et al. 2003, for details of the method); ${ }^{(b)}$ ages and masses computed by us from Palla \& Stahler (1993) tracks using the methods described in Testi et al. (1998); ${ }^{(c)}$ luminosity calculated using bolometric correction.

References: (1) SIMBAD data base; (2) Smith \& Terrile (1984); (3) Barrado y Navascués et al. (1999); (4) Bouret et al. (2002); (5) Crifo et al. (1997); (6) Malfait et al. (1998); (7) Meeus et al. (2001); (8) Dominik et al. (2003); (9) Dent et al. (2005); (10) Meeus et al. (2002); (11) Acke \& Waelkens (2004); (12) van den Ancker et al. (1998); (13) Beskrovnaya et al. (1999); (14) Bertout et al. (1999); (15) Cidale et al. (2001); (16) Mora et al. (2001); (17) Torres et al. (2003); (18) Corcoran \& Ray (1997); (19) Valenti et al. (2000); (20) Böhm \& Catala (1995); (21) Royer et al. (2002); (22) Gerbaldi et al. (1999); (23) Finkenzeller \& Jankovics (1984); (24) Böhm \& Catala (1993); (25) Bouret \& Catala (1998); (26) Lada (1985); (27) Hernández et al. (2004); (28) Dunkin et al. (1997); (29) Donati et al. (1997); (30) Siebenmorgen et al. (2000); (31) Millan-Gabet et al. (2001); (32) Bouret et al. (2003); (33) Miroshnichenko et al. (2001); (34) Martin et al. (2004). 


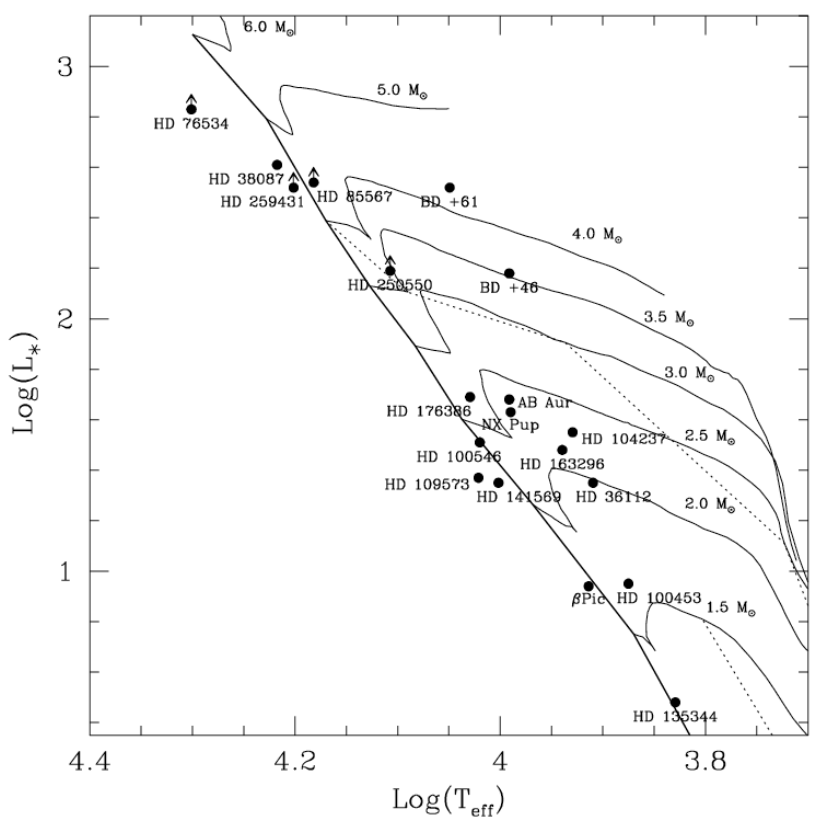

Fig. 1. The stars of our sample are plotted on this HR diagram. The evolutionary tracks between the pre-main sequence phase and the ZAMS are shown for the Ae/B9 stars. For this plot, we used the Palla \& Stahler (1993) pre-main sequence tracks and the interpolation routines written by Testi et al. (1998). For the more massive Be stars, the pre-main sequence stage is less obvious due to the faster evolution of these stars, which makes them appear directly on the ZAMS.

levels of higher vibrational levels $(v \geq 1)$ of the fundamental electronic level can also be observed (e.g. Lecavelier des Etangs et al. 2003; Bouret et al. 2003; Boissé et al. 2005).

For the hottest stars in our sample, typically stars whose $T_{\text {eff }}$ values are greater than $10000 \mathrm{~K}$, the FUV stellar flux is high enough to observe circumstellar and/or interstellar (CS/IS) lines in absorption against the continuum. For the coolest stars, the lack of sufficient flux in the continuum prevents easy detection of CS/IS features. However, as highlighted by Lecavelier des Etangs et al. (2001), stellar emission lines, when present, can provide "continuum" flux for CS/IS absorption features. Most of the cool stars in our sample exhibit broad emission in the O VI $\lambda \lambda 1032-1038$ resonance doublet (Deleuil et al. 2006). A number of $\mathrm{H}_{2}$ transitions occur in the spectral domain near $1034 \AA$. In particular, the strongest transitions of $\mathrm{H}_{2}$, which arise from the $J=0$ to $J=2$ levels, fall in the same wavelength range as the $1038 \AA$ emission line of the O VI doublet. This line is the weaker component of the O VI doublet and, as already shown by Roberge et al. (2001), the flux in the blue part of the emission profile can be completely suppressed by strong $\mathrm{H}_{2}$, but also by C II absorption (Roberge et al. 2006), making detection of $\mathrm{H}_{2}$ challenging. In that case, other lines corresponding to transitions arising from upper $J$-levels should be observed, especially, the spectral lines from transitions arising from the $J=3$ and $J=4$ rotational levels, which fall in the spectral domain of the $1032 \AA$ O VI emission line.

We detected and measured $\mathrm{H}_{2}$ absorption lines in the spectra of all but 6 stars. When $\mathrm{H}_{2}$ is detected, we identified absorption lines of $\mathrm{H}_{2}$ arising from the rotational levels of the ground vibrational state. Transitions arising from rotational levels as high as $J=5$ of the first excited vibrational state $(v=1)$ of the ground electronic level are also detected in some cases. We performed molecular and atomic absorption line analysis using the
OWENS profile fitting procedure written by Lemoine (Lemoine et al. 2002; Hébrard et al. 2002), which allowed us to simultaneously fit all lines of each species. The wavelengths and oscillator strengths of the $\mathrm{H}_{2}$ lines were obtained from Abgrall et al. (1993a) for the Lyman system and Abgrall et al. (1993b) for the Werner system. The inverse of the total radiative lifetimes are reported in Abgrall et al. (2000). For details on the line analysis procedure, we refer the reader to our previous studies (e.g. Bouret et al. 2003; Martin et al. 2004; Martin-Zaïdi et al. 2005). We measured the column densities at each energy level of $\mathrm{H}_{2}$, the radial velocities, and the line widths. For most of the stars, the $\mathrm{H}_{2}$ mean projected velocity is very close to that of the star, within the resolution of the FUSE spectra $\left(\sim 15 \mathrm{~km} \mathrm{~s}^{-1}\right)$. This is a clue that the detected gas is CS in origin. Our results are given in Table 3 with 2- $\sigma$ error bars. Table 4, available as on-line material, contains the following information. Columns 1 and 2 list the number of the vibrational $(v)$ and rotational $(J)$ level, Col. 3 gives the statistical weight of each level, Col. 4 gives the energy of each level, and Cols. 5 to 15 present the column densities of each energy level of $\mathrm{H}_{2}$, when detected, for all the targets stars.

Using the column densities measured for each level, we plotted the excitation diagrams. These diagrams show the ratio of the column density to the statistical weight of each energy level versus the energy of the level. They characterize the excitation conditions of the gas and give information about the temperature of the gas and its physical conditions. For the stars in our sample, the diagrams show marked differences between the Herbig Ae/B9 stars, on the one hand, and the Herbig Be stars, on the other. We distinguished between these two sub-groups of stars in our analysis (see Sects. 4 and 5).

\section{2. $\mathrm{H}_{2}$ modeling}

Absorption line spectroscopy does not allow us to directly measure the distance of the absorbing material along the line of sight. In a first attempt to better diagnose the origin of the detected molecular gas and its excitation conditions, we modeled the CS $\mathrm{H}_{2}$ spectra using a photodissociation region (PDR) model. We used the PhotoDissociation Region code of Le Petit et al. (2006), referred to as the "Meudon PDR Code". The code has been developed as a tool to investigate the physical conditions in both diffuse and dense IS clouds. The physics included in the code, as well as the numerical techniques and several recent improvements, are fully described in Le Petit et al. (2006). We refer the reader to this paper and provide only the main outlines of the code here.

The code models a slab of IS medium, in a stationary state, heated by one star's radiation field and the galactic field in a plane-parallel geometry. The IS radiation field flux is assumed isotropic on both sides of the cloud, while the added incident stellar radiation is normal on one side. The code uses a standard galactic field law (Draine 1978). The stellar FUV radiation field is given by a blackbody law calculated from the star's radius and temperature and the star-to-cloud distance. The model input parameters are thus the radius and effective temperature of the star, the distance of the observed gas from the star, the density of the medium, and the extinction $\left(A_{\mathrm{v}}\right)$. The equations of thermal and chemical balance as a function of depth within the cloud are solved in a plane-parallel geometry with 113 chemical species and 967 chemical reactions included. As a result, the code provides the temperature distribution within the gaseous medium and the abundances of the chemical species derived from the chemical balance. 
Table 3. Total column densities, radial velocities, intrinsic line widths $b$, and origin of the detected $\mathrm{H}_{2}$ towards each star in the sample.

\begin{tabular}{|c|c|c|c|c|c|c|c|}
\hline Name & $\log N\left(\mathrm{H}_{2}\right)^{(a)}$ & $\begin{array}{c}v_{\mathrm{rad}}\left(\mathrm{H}_{2}\right)^{(b)} \\
\left(\mathrm{km} \mathrm{s}^{-1}\right)\end{array}$ & $\begin{array}{c}b \\
\left(\mathrm{~km} \mathrm{~s}^{-1}\right)\end{array}$ & $\begin{array}{l}\text { Origin } \\
\text { of } \mathrm{H}_{2}\end{array}$ & Ref. & $\begin{array}{c}\text { Inclination } \\
\text { of the disk }\left(^{\circ}\right)^{(c)}\end{array}$ & Ref. \\
\hline$\beta$ Pictoris & $\leq 17.41$ & - & - & - & (1) & $\sim 0$ & (6) \\
\hline HD 135344 & $\leq 17$ & - & - & - & (1) & $79 \pm 2$ & (7) \\
\hline HD 100453 & $\leq 16$ & - & - & - & (1) & $\sim 65$ & (8) \\
\hline HD 36112 & $\leq 17.85$ & - & - & - & (1) & $53-57$ & (9) \\
\hline HD 104237 & $18.68_{-0.61}^{+0.39}$ & $1.4_{-1.0}^{+0.9}$ & $5.3_{-0.7}^{+0.6}$ & $\mathrm{CS}$ & (1) & 78 & (10) \\
\hline HD 163296 & $18.16_{-0.40}^{+0.27}$ & $0 \pm 2$ & $2.2_{-0.7}^{+0.5}$ & $\mathrm{CS}$ & (2), (1) & $60 \pm 5$ & (11) \\
\hline NX Pup & $\leq 19.30$ & - & - & - & (1) & $?$ & \\
\hline AB Aur & $20.03_{-0.19}^{+0.15}$ & $2 \pm 3$ & $6.8_{-2.2}^{+2.8}$ & IS/CS? & $(1),(4)$ & $55-63$ & (13) \\
\hline HD 141569 & $20.32_{-0.22}^{+0.20}$ & 20 & $5.3_{-0.5}^{+0.3}$ & IS & (1) & $51 \pm 3$ & (14) \\
\hline HD 100546 & $16.46_{-0.14}^{+0.24}$ & $0 \pm 2$ & $3.4_{-0.5}^{+0.8}$ & $\mathrm{CS}$ & (2), (1) & $51 \pm 3$ & (15) \\
\hline HD 109573 & $\leq 15.40$ & - & - & - & (1) & $20.5 \pm 3$ & (12) \\
\hline HD 176386 & $20.80_{-0.26}^{+0.18}$ & $\sim-7.0$ & & $\mathrm{CS}$ & (1) & $n$ & \\
\hline HD 250550 & $19.26_{-0.40}^{+0.17}$ & $-1.0_{-20}^{+1.7}$ & $5.6_{-0.8}^{+0.7}$ & $\mathrm{CS}$ & (3) & $n$ & \\
\hline HD 85567 & $19.33_{-0.17}^{+0.20}$ & $4.5_{-0.2}^{+0.3}$ & $6.0_{-0.9}^{+0.7}$ & $\mathrm{CS}$ & (1) & $n$ & \\
\hline HD 259431 & $20.64_{-0.19}^{+0.11}$ & $13.0_{-1.8}^{+2.2}$ & $4.5_{-0.5}^{+0.3}$ & $\mathrm{CS}$ & (3) & $n$ & \\
\hline HD 38087 & $20.43_{-0.08}^{+0.15}$ & $2.36_{-2.1}^{+1.6}$ & $3.7_{-0.2}^{+0.3}$ & $\mathrm{CS}$ & (1) & $n$ & \\
\hline HD 76534 & $20.64_{-0.16}^{+0.16}$ & $0.0_{-1.3}^{+0.3}$ & $5.0_{-0.3}^{+0.4}$ & $\mathrm{CS}$ & (5) & $n$ & \\
\hline
\end{tabular}

(a) Upper limits on the column densities have been obtained with $b=1 \mathrm{~km} \mathrm{~s}^{-1}$.

(b) Radial velocities are in the stars' rest frames.

(c) The inclination angles for the disks are given with respect to the line of sight ( $i=0$ : edge-on). " $n$ " indicates stars with no evidence of disk.

References in Col. 6 concern the results given in Cols. 2 to 5, and references in Col. 8 are for the inclination angles of the disks. References: (1) this work; (2) Lecavelier des Etangs et al. (2003); (3) Bouret et al. (2003); (4) Roberge et al. (2001); (5) Martin et al. (2004); (6) Smith \& Terrile (1984); (7) Dent et al. (2005); (8) Dominik et al. (2003); (9) Eisner et al. (2004); (10) Grady et al. (2004); (11) Grady et al. (2000); (12) Augereau et al. (1999); (13) Eisner et al. (2003); (14) Weinberger et al. (1999); (15) Augereau et al. (2001).

Without any precise information on the nature of the dust grains in the absorbing medium, we used standard ISM values for the grain size distribution and properties (Mathis et al. 1977). When available, we used IUE spectra to determine the column density of HI, which allowed us to estimate the ratio of the gas column to the $A_{\mathrm{v}}$ as an input parameter. We used the standard ISM value of this ratio when no observational constraints were available. For most of our targets, little or no information is available in the literature about the properties of the observed gas, namely its distance to the star or its density and temperature. We thus proceeded by an iterative method, varying both the distance and density of the gas to obtain the best fits to the observed excitation diagrams. We checked that the model output parameters are consistent with the molecular fraction we derived from our spectral analysis (see Sect. 5). We also compared the synthetic spectra provided by the code to the observed ones, as an additional check. The results of our modeling are presented in the next sections.

\section{Ae/B9 stars}

In this section, we first present the stars for which we have not detected $\mathrm{H}_{2}$ absorption lines. In the following subsections, when $\mathrm{H}_{2}$ is detected, we distinguished the stars for which we observed cold $\mathrm{H}_{2}$ and warm $\mathrm{H}_{2}$ on the basis of their excitation diagrams.

\subsection{Stars lacking $\mathrm{H}_{2}$ absorption lines}

We could not detect any $\mathrm{H}_{2}$ absorption lines for 6 stars in our sample.

$\beta$ Pictoris: Early FUSE observations of $\beta$ Pictoris in 2000 allowed us to report a clear deficiency of molecular hydrogen in the disk of the star, consistent with the evolved status of the system (Lecavelier des Etangs et al. 2001). Taking advantage of a third exposure acquired in November 2002 with a total exposure time of about $50000 \mathrm{~s}$, much longer than the two previous ones, we checked for any $\mathrm{H}_{2}$ absorption features in the new spectra (Fig. 2). Co-adding all three spectra allowed us to refine the upper limit on the total $\mathrm{H}_{2}$ column density previously estimated at about $10^{18} \mathrm{~cm}^{-2}$ by Lecavelier des Etangs et al. (2001). Assuming a low value for the intrinsic line width $\left(b=1 \mathrm{~km} \mathrm{~s}^{-1}\right)$, we found the new estimate to be less than $2.6 \times 10^{17} \mathrm{~cm}^{-2}$.

HD 109573: HD 109573 is the only star in our study for which no $\mathrm{H}_{2}$ absorption lines could be detected, despite a noticeable stellar continuum flux. The stellar flux is well-developed in the reddest part of the FUSE wavelength range, above $1110 \AA$. Below this wavelength, where the strongest $\mathrm{H}_{2}$ transitions fall, the stellar flux level is much lower but would be high enough to observe $\mathrm{H}_{2}$ absorption lines if present, but the signal-to-noise ratio is very low $(S / N \simeq 1.5)$ and no stellar emission lines are present to overcome this issue.

HD 109573 is also an evolved system, the star being very close to the main sequence. It is known to possess a flat debris disk seen nearly edge-on, with an inclination angle of about $20^{\circ}$ from the line of sight (Augereau et al. 1999). The dusty disk has two components: an inner annulus that extends between 4-9 $\mathrm{AU}$ to $\sim 55 \mathrm{AU}$ from the central star, and an outer ring at $70 \mathrm{AU}$ (Wahhaj et al. 2005; Augereau et al. 1999). These two components are separated by a gap located between 55 and $60 \mathrm{AU}$ from the star, which is probably the consequence of planet formation (Augereau et al. 1999). The outer ring is about $15 \mathrm{AU}$ thick, with an inclination angle of $13 \pm 1^{\circ}$ from the 


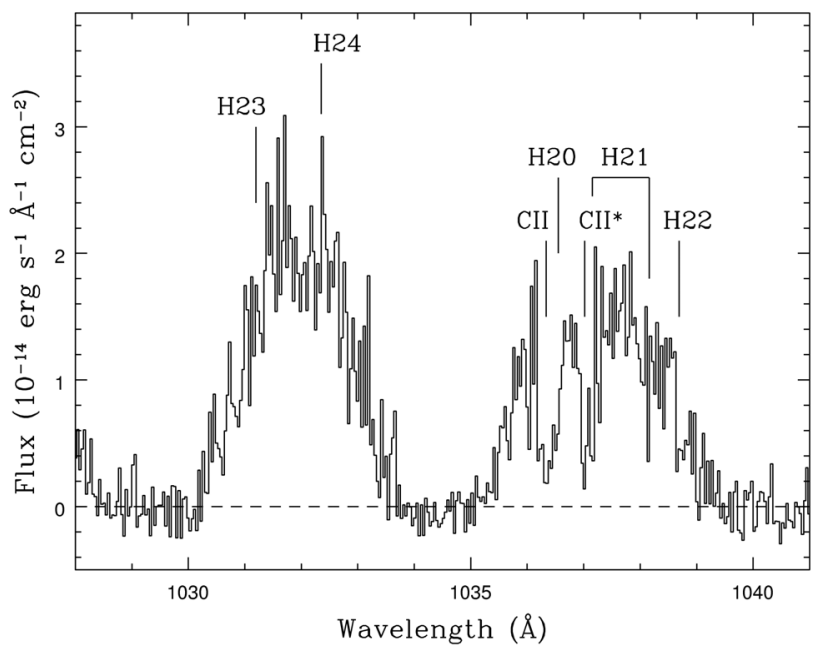

Fig. 2. O VI emission doublet near $1032 \AA$ and $1038 \AA$ in the FUSE spectrum of $\beta$ Pictoris observed in 2002. Here, only the exposures obtained during the night have been co-added, to avoid contamination by strong airglow emission lines due to the Earth's atmosphere. The positions of the $\mathrm{H}_{2}$ transitions we should observe if $\mathrm{H}_{2}$ was present along the line of sight are plotted. The $\mathrm{H}_{2}$ levels are marked $\mathrm{H} 20$ for $(v=0, J=0), \mathrm{H} 21$ for $(v=0, J=1)$, etc. For details on the $\mathrm{C}$ II absorption, see Roberge et al. (2006).

line of sight (Telesco et al. 2000). This implies that the upper layers of the disk intersect the line of sight and could potentially produce absorption features. However, as previously reported by Chen \& Kamp (2004), we do not observe absorption lines of $\mathrm{H}_{2}$ in the FUSE spectrum. We set an upper limit on the total column density of $\mathrm{H}_{2}$ of $\leq 2.5 \times 10^{15} \mathrm{~cm}^{-2}$, which is consistent with the limit found by Chen \& Kamp (2004). This non-detection of $\mathrm{H}_{2}$ implies that the disk of HD 109573 is almost depleted of molecular gas, which agrees with the evolutionary status of this star.

NX Pup, HD 36112, HD 135344, and HD 100453: The photospheric continuum of these four stars is too low in the FUV $(S / N \sim 2$ at $1100 \AA)$ for a reliable detection of CS/IS absorption lines. However, as in the case of $\beta$ Pictoris, we took advantage of the OVI emission lines to place limits on the total $\mathrm{H}_{2}$ column densities along the line of sight. The presence of a CS disk around NX Pup is suspected from its SED. For the three other stars, all younger than $\beta$ Pictoris, the non-detection of $\mathrm{H}_{2}$ is consistent with the high inclination angles for their disks, which do not intersect the lines of sight to the stars (see Table 2). It shows that, if present, the CS gas does not extend far enough above the midplanes of the disks to be detected in absorption and that the remnant of the initial CS envelopes have been dispersed or are too low in density to allow detection.

\subsection{Cold $\mathrm{H}_{2}$}

HD 141569: The HD 141569 disk is usually called a transitional disk, in which there are signs of dust clearing in the inner disk, but significant primordial molecular gas is still supposed to be present in the disk. In a previous detailed analysis (see Martin-Zaïdi et al. 2005), we demonstrated that the absorption lines of molecular hydrogen and several atomic species observed in its FUSE spectrum are very likely related to the slightly reddened diffuse outer region surrounding the L134N dark cloud complex (Juvela et al. 2002). The non-detection of CS gas implies that there is no remnant CS envelope and that all the gas has had time to collapse into a flat or very slightly flared

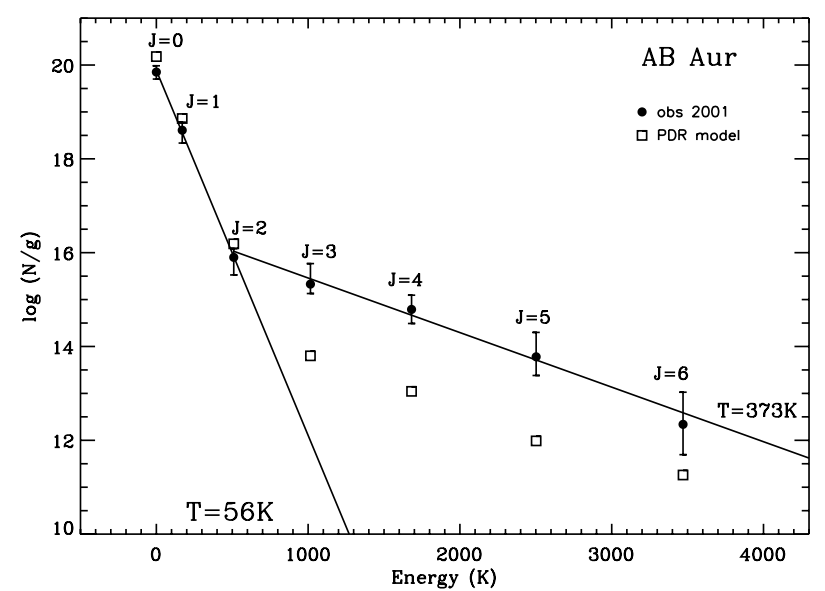

Fig. 3. Excitation diagram for $\mathrm{H}_{2}$ towards $\mathrm{AB}$ Aurigæ. The observed level column densities (filled circles) show that $\mathrm{H}_{2}$ is thermalized up to $J=2$ with a low kinetic temperature of about $56 \mathrm{~K}$, while the column densities of the higher $J$-levels are consistent with a temperature of about $373 \mathrm{~K}$. The populations of the first three energy levels are reproduced well by a PDR model (open square) with a low density of about $250 \mathrm{~cm}^{-3}$ and a distance between the star and the gas of about $0.3 \mathrm{pc}$, with an excitation temperature of about $55 \mathrm{~K}$. Our model does not reproduce the higher energy levels' excitation. This may be due to the presence of a second warm/hot gaseous component along our line of sight (see text).

disk. This result is consistent with the ${ }^{12} \mathrm{CO}$ observations at 345.796 GHz described in Dent et al. (2005).

AB Auriga: The presence of $\mathrm{H}_{2}$ in the far-UV spectra of AB Aurigæ was first reported and analyzed by Roberge et al. (2001) from an early FUSE observation. A new observation obtained more than one year later allows us to confirm and refine the analysis.

We identified and measured $\mathrm{H}_{2}$ lines arising from $J=0$ to $J=6$ in the ground vibrational state $(v=0)$. As suggested by Roberge et al. (2001), the $\mathrm{H}_{2}$ could be IS in origin or have a CS origin, as a cold remnant of its natal cloud or a CS envelope (Semenov et al. 2005). The relatively low spectral resolution of FUSE prevents us from drawing firm conclusions about the origin of the detected gas on the basis of its radial velocity and line width. The excitation diagram derived from the column densities in the $J$-levels may provide additional clues for further constraining the location of the observed gas. For AB Aur, it confirms that the $\mathrm{H}_{2}$ is thermalized up to $J=2$ with a low kinetic temperature of $56 \pm 4 \mathrm{~K}$ (Fig. 3), results in good agreement with those of Roberge et al. (2001). Since the relative populations of the lower $\mathrm{H}_{2}$ energy levels are determined primarily by thermal collisions, the excitation temperature found from these levels should be close to the kinetic temperature of the gas. On the other hand, the column densities of the higher $J$-levels are compatible with thermal equilibrium at a temperature of $373 \pm 83 \mathrm{~K}$.

Using the Meudon PDR Code, we checked that such excitation conditions could not be reproduced by a single absorbing component along the line of sight towards the star. We varied the different parameters within their error bars and tried to model the observations with different $A_{\mathrm{v}}$ values found in the literature, but no PDR model fit the data. There are still some free parameters to explore, but we need additional observational constaints to make an accurate modeling. As shown in Fig. 3, a diffuse component $\left(n_{\mathrm{H}} \sim 250 \mathrm{~cm}^{-3}\right)$ located at $0.3 \mathrm{pc}$ from the star reproduces the column densities of the three first energy levels, but failed to reproduce the higher $J$-level populations. 
Two-temperature behavior in excitation diagrams for $\mathrm{H}_{2}$ is often observed in diffuse and translucent IS clouds (e.g. Spitzer \& Cochran 1973; Gry et al. 2002; Browning et al. 2003), but its origin is not understood well and remains a subject of controversy, as reviewed and discussed in Snow \& McCall (2006). Several excitation mechanisms could be invoked separately or all together to explain this behavior. Shull \& Beckwith (1982) initially suggested that the populations of the higher levels can be inflated by UV and/or formation pumping and radiative cascades. We checked that, to populate the $J>2 \mathrm{lev}$ els by UV pumping alone, we would have to increase the radiation field ( $G \sim 1.5$ in Draine's units) by a factor of more than 10. An alternative model invokes local heating of the gas within the cloud. Such heating could be generated by magnetohydrodynamic shocks (see Chieze et al. 1998) or transient smallscale turbulence that results in localized tiny warm regions, as suggested by Falgarone \& Puget (1995).

The simplest explanation is, however, the presence of distinct absorbing media along the line of sight with very different excitation conditions (Browning et al. 2003). For all the stars in this study, we found $b$-values much higher than expected for pure thermal broadening. This is an unusual situation in diffuse ISM clouds, where typical $b$-values are about 1.5 up to $3 \mathrm{~km} \mathrm{~s}^{-1}$ (e.g. Gry et al. 2002). However, higher values are also routinely reported (e.g. Lehner et al. 2003; Redfield \& Linsky 2004). The simplest explanation for this phenomenon is the presence of several gaseous components along the line of sight that could appear blended in the spectra. But it could also be related to gas motions on different spatial scales as suggested by different studies. Such motions could originate in turbulent and warm layers within the cloud, through transient shocks or vortices (Joulain et al. 1998; Gredel et al. 2002), which lead to increased observed $b$-values. Without observations at higher spectral resolution or additional constraints on the chemistry of the gaseous medium, we cannot distinguish between explanations.

In conclusion, we cannot distinguish with certainty yet whether the $\mathrm{H}_{2}$ gas in this source has a circumstellar or interstellar origin, but the cold component appears to be located at a relatively large distance from the star $(\sim 0.3 \mathrm{pc})$.

Now the question arises, whether the gas has a circumstellar origin, i.e. could it be part of the disk or must it be part of a separate, more spherically distributed, components of CS gas? $\mathrm{AB}$ Aur is known to harbor an extended CS disk with an inclination angle between $27^{\circ}$ and $35^{\circ}$ from the plane of the sky (Eisner et al. 2003; Pantin et al. 2005), i.e. nearly face-on. Since a detection of $\mathrm{H}_{2}$ in absorption means that the line of sight toward the star must pass through the $\mathrm{H}_{2}$ gas, the near-face-on inclination appears to make it unlikely that the gas is part of the disk. However, a more quantitative analysis is required to make any firm statements. Suppose the disk is at its thickest (geometrically) at a radius $r$, where it has a gas surface density $\Sigma_{\text {gas }}$ and a gas temperature $T$. Assuming vertical hydrostatic equilibrium, the gas density as a function of height $z$ above the midplane at that radius is

$\rho_{\text {gas }}=\frac{\Sigma_{\text {gas }}}{\sqrt{2 \pi} H_{\mathrm{p}}} \exp \left(-\frac{z^{2}}{2 H_{\mathrm{p}}^{2}}\right)$

with

$H_{\mathrm{p}}=\sqrt{\frac{k T r^{3}}{\mu m_{\mathrm{p}} G M_{*}}}$,

where $\mu=2.3$ is the mean molecular weight of the molecular-hydrogen-helium mixture, $m_{\mathrm{p}}$ the proton mass, $G$ the gravitational constant, and $k$ the Boltzmann constant. The column depth along the line of sight passing through $(r, z)$, i.e. with the disk at an inclination of $i=\arctan (r / z)$ away from face-on, is then estimated as

$N_{\mathrm{H}_{2}}=\frac{\rho_{\mathrm{gas}} l}{\mu m_{\mathrm{p}}} \simeq \frac{\rho_{\mathrm{gas}} H_{\mathrm{p}}}{\mu m_{\mathrm{p}}}$.

Here we simply took as radial extent $l$ the pressure scale height $l \simeq H_{\mathrm{p}}$. In reality this may be a factor of a few higher.

Let us take $r$ to be the outer radius of the disk estimated at $r_{\text {out }}=800 \mathrm{AU}$ (Semenov et al. 2005) and a disk mass of at most $1 M_{\odot}$. With $M_{\text {disk }} \simeq \Sigma_{\text {gas }}\left(r_{\text {out }}\right) \pi r_{\text {out }}^{2}$, this gives a surface density of about $\Sigma_{\text {gas }} \simeq 4 \mathrm{~g} / \mathrm{cm}^{2}$ at $r=r_{\text {out }}$. Using these numbers and the stellar parameters from Table 2, we find $H_{\mathrm{p}}\left(r_{\text {out }}\right) / r_{\text {out }}=0.36$ for a temperature of $T=95 \mathrm{~K}$, which, with an inclination of $35^{\circ}$ away from face-on, yields a column density of $\log N\left(\mathrm{H}_{2}\right)=20$ as given in Table 3. In other words: we need a temperature of about $95 \mathrm{~K}$ to puff the disk up enough for sufficient matter to cross the line of sight at around 800 AU distance. Considering the roughness of this estimate and the closeness to the excitation temperature of $56 \mathrm{~K}$ for the low-lying levels, it shows that it is possible in principle that this gas is part of the outer part of a massive disk. But the same derivation yields a volume density of $n_{\mathrm{H}_{2}} \simeq 3.4 \times 10^{4}$, which is much higher than the $\sim 250 \mathrm{de}-$ rived from the PDR model. Instead of $800 \mathrm{AU}$, we could also use $0.3 \mathrm{pc} \equiv 61900 \mathrm{AU}$ as reference radius $r$. Then any estimate would yield the disk as gravitationally unbound, and we cannot speak of a "disk". Thus, if the cool material is indeed located at such a distance, it may still be associated with the star AB Aurigae, but it would be meaningless to speak of disk material there and is likely to be either a remnant envelope or outflowing material from the disk.

\subsection{Warm $\mathrm{H}_{2}$ gas}

We re-did the spectral analyses for HD 100546 and HD 163296, which were presented in Lecavelier des Etangs et al. (2003), and found the same results. We also refined the analysis of the FUSE spectrum of HD 104237 presented in Herczeg et al. (2003) and found compatible results. In the HD 100546 spectrum, lines arising from the $J$-levels of the ground vibrational level $(v=0)$ up to $J=10$ and $J$-levels of the first vibrational level $(v=1)$ up to $J=5$ are detected. In the HD 163296 and HD 104237 spectra, only lines arising from the $J$-levels up to $v=0, J=4$ and $v=0, J=5$, respectively, are observed. Higher rotational $J$-levels and vibrational levels are beyond the detection limit, because of the sharp flux decrease with decreasing wavelength caused by the later spectral types of these two stars and because of the lower signal-to-noise ratios of the data. These low signal-to-noise ratios are responsible for the rather large uncertainties in the determinations of the column densities (Fig. 4).

We plotted the excitation diagrams of $\mathrm{H}_{2}$ for each of the three stars, and found two-temperature behavior in the case of HD 100546 (Fig. 4). In contrast to the sources in Sect. 4.2, for all three stars, the $\mathrm{H}_{2}$ is thermalized up to $J=4$ with surprisingly high kinetic temperatures, greater than $300 \mathrm{~K}$ and as high as $758 \mathrm{~K}$ in the case of HD 100546 (Fig. 4). In addition, the temperature derived for the higher $J$-levels observed in HD 100546, is $1495 \pm 70 \mathrm{~K}$. Such excitation conditions clearly differ from those observed in the interstellar medium. In addition, the measured radial velocities favor a CS origin for the $\mathrm{H}_{2}$ at the FUSE spectral resolution. 

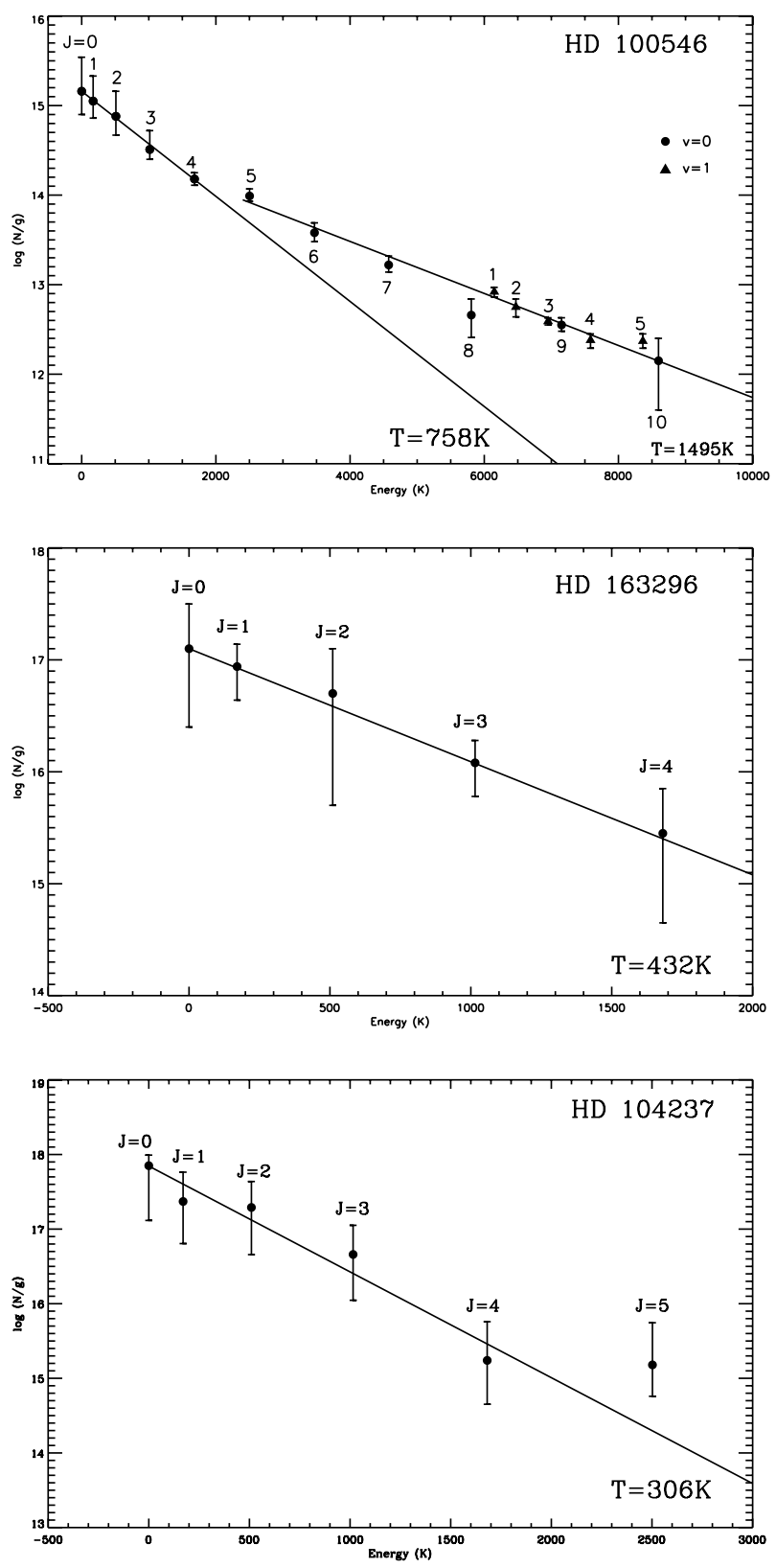

Fig. 4. a) Excitation diagram of $\mathrm{H}_{2}$ for $\mathrm{HD} 100546$. The $\mathrm{H}_{2}$ is thermalized up to $J=4$ with a kinetic temperature of $758 \pm 147 \mathrm{~K}$. The column densities of the higher $J$-levels are consistent with a temperature of about $1495 \pm 70 \mathrm{~K}$. b) Excitation diagram of $\mathrm{H}_{2}$ for $\mathrm{HD} 163296$. As for $\mathrm{HD} 100546$, the $\mathrm{H}_{2}$ is thermalized up to $J=4$ with a lower kinetic temperature of $432 \pm 135 \mathrm{~K}$. Here, only the $v=0$ levels up to $J=4$ are observed because of the later type of the star (A1Ve) and the low $S / N$ ratio of the spectrum. c) Excitation diagram of $\mathrm{H}_{2}$ for $\mathrm{HD} 104237$. The $\mathrm{H}_{2}$ is thermalized up to $J=4$ with a kinetic temperature of $306 \pm$ $80 \mathrm{~K}$. The higher $J$-levels may be consistent with a higher temperature, as suggested by the $J=5$ column density.

Using the numerical model developed by Le Bourlot et al. (1993), we estimated that the warm $\mathrm{H}_{2}$ (with $J \leq 4$ ) should be located at a distance of about 1.5 AU from HD 100546, 4 AU from HD 163296, and 9.5 AU from HD 104237 to explain the observed excitation conditions (for details about the method see Lecavelier des Etangs et al. 2003). The observed temperature cannot be explained by the Galactic UV radiation field, which implies that the observed $\mathrm{H}_{2}$ is circumstellar and lies very close to its central star. Using the volume densities derived from the critical density given by Le Bourlot et al. (1999), we found typical thicknesses of $\leq 0.2 \mathrm{AU}$ for the absorbing layer towards HD 100546, $\leq 1.2$ AU for HD 163296 and $3.2 \mathrm{AU}$ for HD 104237. These results reinforce our interpretation of a CS origin for the detected gas.

This again poses the question of whether this material is part of the disk or part of some more spherically distributed gaseous circumstellar material. For all three sources, the inclination is more than $50^{\circ}$ away from edge-on. Using Eq. (3) with a gas surface density of, say, $\Sigma_{\text {gas }}=10^{4} \mathrm{~g} / \mathrm{cm}^{2}$, at the radii estimated above, and inclination of $50^{\circ}$ and required column densities given in Table 2, we find that we need a temperature of $11600 \mathrm{~K}$ for HD 100546, $4700 \mathrm{~K}$ for HD 163296, and $2300 \mathrm{~K}$ for HD 104237 to support hydrostatic equilibrium and yet have the required column density of $\mathrm{H}_{2}$. All these temperatures are well above the excitation temperatures measured for these sources, so the disk is excluded as the carrier of the warm $\mathrm{H}_{2}$ gas we detected.

We can think of two possibilities to explain the excitation of this gas. The first is that this medium is related to a nonradiatively heated medium close to the stellar surface, such as a chromosphere, as proved by the well-known FUV and X-ray activity of these stars (e.g. Deleuil et al. 2004, 2005; Grady et al. 2004). Others Herbig Ae stars exhibit noticeable activity in the far-UV but at a much lower level (Deleuil et al. 2006). The $\mathrm{H}_{2}$ we observe could be produced in the very outer layer of this extended hot region. Due to the inclination angle of the disk, this region should have a more or less spherical geometry to allow gas to be observed in absorption.

Another natural origin for the excited $\mathrm{H}_{2}$ is photoevaporation of the disk by FUV stellar radiation (Hollenbach private communication). This is a mechanism of disk dissipation similar to the better known EUV-driven photoevaporation (e.g. Hollenbach et al. 1994), but this time driven by non-ionizing FUV photons. In contrast to the ionized EUV-driven wind, which originates as close as $\sim 1$ AU from the star, the FUV-driven wind is neutral and originates in the outer regions of the disk (many tens of AU away from the star). It is expected to have a much stronger wind mass loss rate than the EUV wind. According to Gorti \& Hollenbach (ApJ submitted), such strong disk winds are expected to flow away from the source in a nearly spherical way, and they are therefore interesting candidates for explaining the observed warm circumstellar matter. The problem in this case is, however, that this FUV driven wind originates much farther out than the few AU radii derived from the excitation diagrams.

Further observations, such as IR spectra of $\mathrm{H}_{2}$, are required to give a better understanding of the origin of the gas and the physical processes that produce this excited $\mathrm{H}_{2}$. More modeling of photoevaporating gas or chromosphere models, which would take non-LTE excitation mechanisms into account, could also help to better constrain the excitation conditions.

\section{Be stars}

This second group of stars contains all the stars earlier than B9 type, which are also the youngest stars in the sample. For all but one, namely HD 250550, we have identified and measured lines arising from the rotational levels of the ground vibrational level, up to at least $J=7$, and also lines from the first vibrational level. The derived excitation diagrams, plotted in Fig. 5, show that the $\mathrm{H}_{2}$ is thermalized up to $J=3$ with a kinetic temperature around $100 \mathrm{~K}$. The temperatures given by the column densities of the high $J$-levels range from $\sim 500 \mathrm{~K}$ to $\sim 1600 \mathrm{~K}$. The radial velocities of this matter suggests that it is of circumstellar origin.

As seen in Fig. 5, the excitation diagrams point to similar excitation conditions for all the stars, which is a clue to a common 

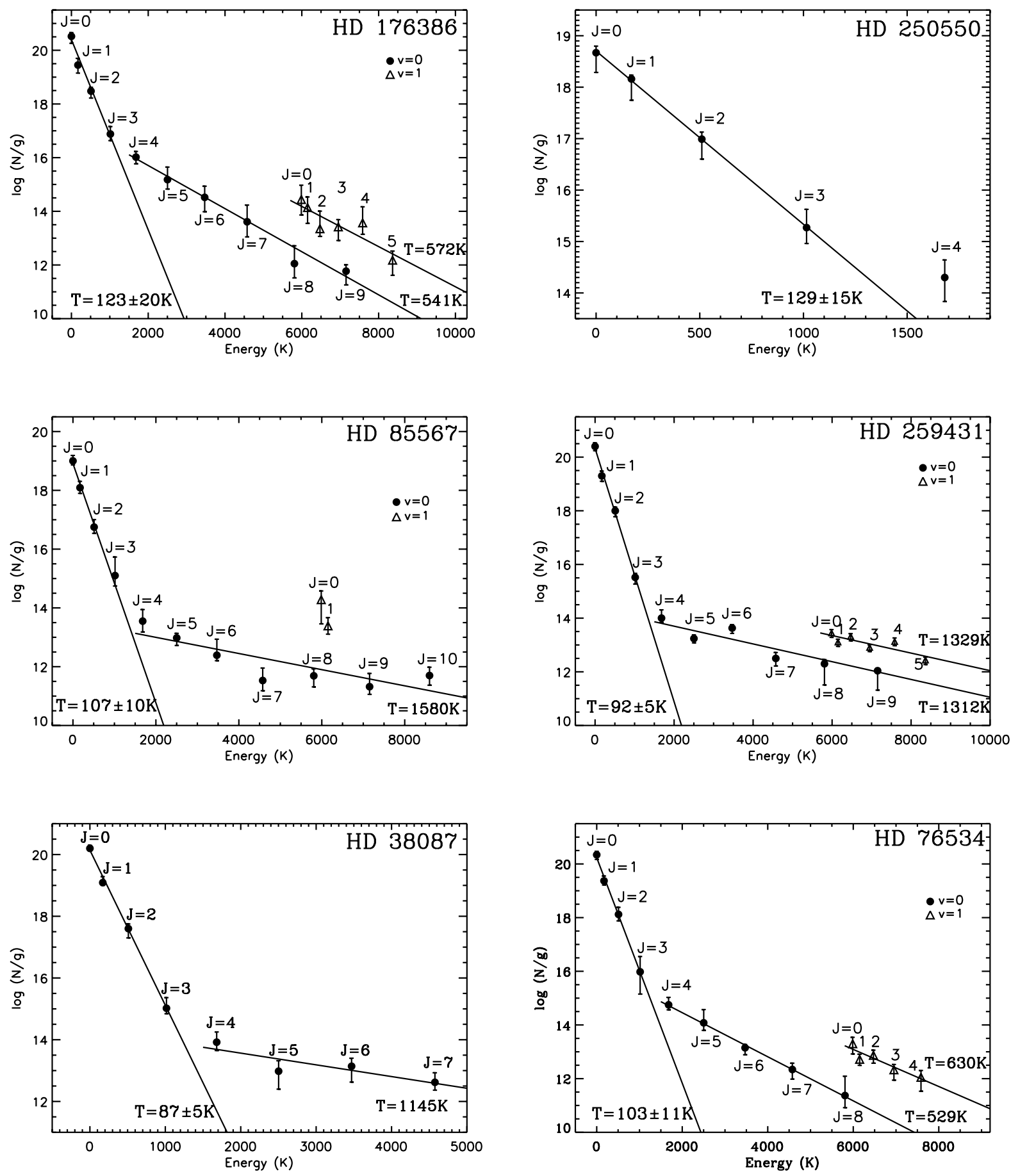

Fig. 5. Excitation diagram for $\mathrm{H}_{2}$ towards the Be stars in our sample. For each star, the $\mathrm{H}_{2}$ is thermalized up to $J \geq 3$ with a kinetic temperature of about $\sim 100 \mathrm{~K}$.

structure in their CS environments. Moreover, since the $v \sin i$ of these stars span a wide range of values (see Table 2) and since the detection of $\mathrm{H}_{2}$ requires the line-of-sight to go through the material, it is unlikely that the material in all these objects is part of a circumstellar disk. Instead it is more likely that this matter is part of the remnants of the clouds within which the stars were formed.

\subsection{Modeling the $\mathrm{H}_{2}$ excitation}

Little is known about the CS environments of these young stars and to model the physical conditions of the gas we detected, we had to estimate realistic values for the input parameters. To derive the H I column density for each star, we used archival $I U E$ spectra to model the Ly $\alpha$ line (Table 5), when good data were available. Using the $E(B-V)$ values listed in Table 2, we calculated the gas-to-dust ratios $N\left(\mathrm{H}_{\text {tot }}\right) / E(B-V)$, in the environments of the stars and the molecular fractions $(f)$, i.e. the fraction of hydrogen atoms in molecular form (Table 5). Our gas-to-dust ratios values are comparable to the mean interstellar value of about $5.8 \times 10^{21}$ atoms cm $\mathrm{cmag}^{-1}$, as measured for standard IS clouds within $2 \mathrm{kpc}$ of the Sun (Bohlin et al. 1978). This favors an IS origin for the $\mathrm{H}_{2}$ we observed towards these stars. 
Table 5. Column densities of H I measured from the IUE spectra, when good data were available.

\begin{tabular}{|c|c|c|c|}
\hline Stars & $\begin{array}{l}\text { Column density } \\
\text { of } \mathrm{HI}\left(\mathrm{cm}^{-2}\right)\end{array}$ & $\begin{array}{c}N\left(\mathrm{H}_{\text {tot }}\right) / E(B-V) \\
\left(\text { atoms cm }{ }^{-2} \mathrm{mag}^{-1}\right)\end{array}$ & $f$ \\
\hline HD 250550 & $9.00_{-4.0}^{+5.0} \times 10^{20}$ & $4.26_{-1.92}^{+2.33} \times 10^{21}$ & $0.04_{-0.03}^{+0.06}$ \\
\hline HD 259431 & $1.60_{-0.6}^{+2.4} \times 10^{21}$ & $9.51_{-3.49}^{+10.2} \times 10^{21}$ & $0.35_{-0.24}^{+0.37}$ \\
\hline HD 38087 & $1.91_{-0.41}^{+2.09} \times 10^{21}$ & $8.46_{-1.41}^{+7.9} \times 10^{21}$ & $0.22_{-0.13}^{+0.15}$ \\
\hline HD 76534 & $2.40_{-1.4}^{+1.6} \times 10^{21}$ & $1.25_{-0.63}^{+0.77} \times 10^{22}$ & $0.27_{-0.16}^{+0.51}$ \\
\hline
\end{tabular}

(1) Bouret et al. (2003).

Table 6. Distance of the gas from the central star and total density of the observed medium, as determined by the best-fitting model using the Meudon PDR Code.

\begin{tabular}{lcc}
\hline \hline Star & $\begin{array}{c}\text { Gas to star } \\
\text { distance }(\mathrm{pc})\end{array}$ & $\begin{array}{c}\text { Gas volume } \\
\text { density, } n_{\mathrm{H}}\left(\mathrm{cm}^{-3}\right)\end{array}$ \\
\hline HD 176386 & 0.04 & 3000 \\
HD 250550 & 0.03 & 600 \\
HD 85567 & 0.10 & 250 \\
HD 259431 & 0.35 & 70 \\
HD 38087 & 1.60 & 12 \\
HD 76534 & 0.40 & 170 \\
\hline
\end{tabular}

For each star, we run grids of models using the Meudon PDR Code, with different distances and densities for the gas, to find the best-fitting model that is consistent with the observed values of the excitation temperature and molecular fraction. The best-fitting model allowed us to derive the total density and the distance of the gas from the central star, which are not constrained by absorption lines observations. Our results are tabulated in Table 6.

As shown in Fig. 6, the general shape of the excitation diagrams is reproduced well by PDR models with a single component, but some details remain. The temperatures on the plots correspond to the excitation temperatures of the low energy levels $(J=0-3)$ given by the best-fitting models. These temperatures are fully consistent with those observed, and always fall within the error bars of the observed excitation temperatures (Fig. 5). A relatively diffuse and cold component reproduces the excitation of the lower $J$-levels, which includes more than $90 \%$ of the detected gas. However, we emphasize that, as for the HAe stars, our modeling fails to reproduce perfectly the excitation of the first $J$-levels of the ground vibrational level and the higher $J$-levels $(J \geq 6, v=0$ and $J, v=1)$ simultaneously. By increasing the FUV field, one could expect to increase the excitation of these levels, but this would affect the column densities of the lower $J$-levels due to photodissociation. This shows that UV pumping and formation pumping are probably not the only mechanisms playing a role in the excitation of intermediate $J$-levels we observe (see Sect. 3). However, at the present time, the $\mathrm{H}_{2}$ pumping formation process is not completely understood, and one could expect that a slightly different modeling could vary the fit.

Using the photospheric models (see Sect. 2 and Bouret et al. 2003) and the attenuation of the UV continuum provided by the PDR code, we computed the synthetic spectrum of each star. To calculate the spectrum, we only took the first three $J$-levels of $\mathrm{H}_{2}$ into account, which produce the strongest absorption lines. Figure 7 shows the synthetic spectrum of HD 76534 overplotted on the observed FUSE spectrum. The synthetic spectra of the other stars are available on-line (Figs. 8 to 12). The good agreement between the modeled and the observed spectra strengthens confidence in our estimates of the distance, density, molecular fraction, and gas-to-dust ratio we estimated for each star from the different observational data.

\subsection{Additional information on the targets}

- HD 176386 - This star is the primary star of a binary system (Proust et al. 1981; Vaz et al. 1998). Our analysis of the FUV spectrum of the star is consistent with the star being surrounded by a large CS envelope $(d=0.04 \mathrm{pc})$, a result in agreement with previous studies in different spectral domains. Siebenmorgen et al. (2000) showed that the FWHM derived from the ISOCAM observations are indicative of a large extended halo around HD 176386, which was confirmed by the ISOPHOT multi-aperture sequence at $7.3 \mu \mathrm{m}$.

- HD 250550 - In Bouret et al. (2003), we showed that the properties of the $\mathrm{H}_{2}$ and the atomic species derived from the FUSE spectrum of HD 250550 indicate that we are probing a dense CS environment, related to the remnant of the molecular cloud that collapsed to form the star. We refer the reader to this paper for more details.

- HD 85567 - Although our analysis does not allow us to rule out the presence of a CS disk, it does favor an interpretation in terms of a large CS envelope $(d=0.1 \mathrm{pc})$. The presence of two components with different temperatures in the excitation diagram agrees with the Malfait et al. (1998) study, which fit the SED of this star with a two-component, optically-thin, dusty envelope model. The apparent dip in the SED of the star between 6 and $10 \mu \mathrm{m}$ was interpreted as a physical hole in the dust distribution, caused by the break-up of an optically thin dusty disk (Lada \& Adams 1992). In addition, Miroshnichenko et al. (2001) have shown that, without imaging observations, it is impossible to distinguish between an extended, optically-thin, mostly spherical component, and a more compact disk. Those authors show that the emissionline profiles indicate complex structure in the CS envelope, which is likely non-spherical, with an optically-thick component and the possible presence of an extended optically-thin dusty component. We emphasize that if two components are present, we cannot distinguish them at the FUSE resolution.

- HD 259431 - Our analysis, presented in a previous paper (Bouret et al. 2003), indicates that the material we observe is most likely related to a large CS envelope. This is consistent with the study by Malfait et al. (1998), who fit the SED of this star with a two-component, optically-thin, dusty envelope model. Although the mid-IR observations of HD 259431 by Polomski et al. (2002) are consistent with a model of a moderately flared CS disk, there is no clear observational evidence of a disk around this star.

- HD 38087 - Snow \& Witt (1989) concluded from their analysis of the IUE spectrum that the star is surrounded by a dense envelope in which unusual grain growth has occurred. These results were confirmed by Burgh et al. (2002), who show that the region surrounding HD 38087 is dense and that the dust grains may be larger than average in the large nebula (NGC 2024) in which the star is located. From our modeling, we find a very low gas density and a large gas-to-star distance. The $\mathrm{H}_{2}$ we observe in the FUSE spectrum is likely located in the nebula NGC 2024, but the resolution of our observations does not allow us to rule out the presence of a second component corresponding to the star's envelope/disk. - HD 76534 - From a previous analysis (Martin et al. 2004), we concluded that, in the hostile CS environment of HD 76534, which is a B2 star, the presence of a CS disk is 

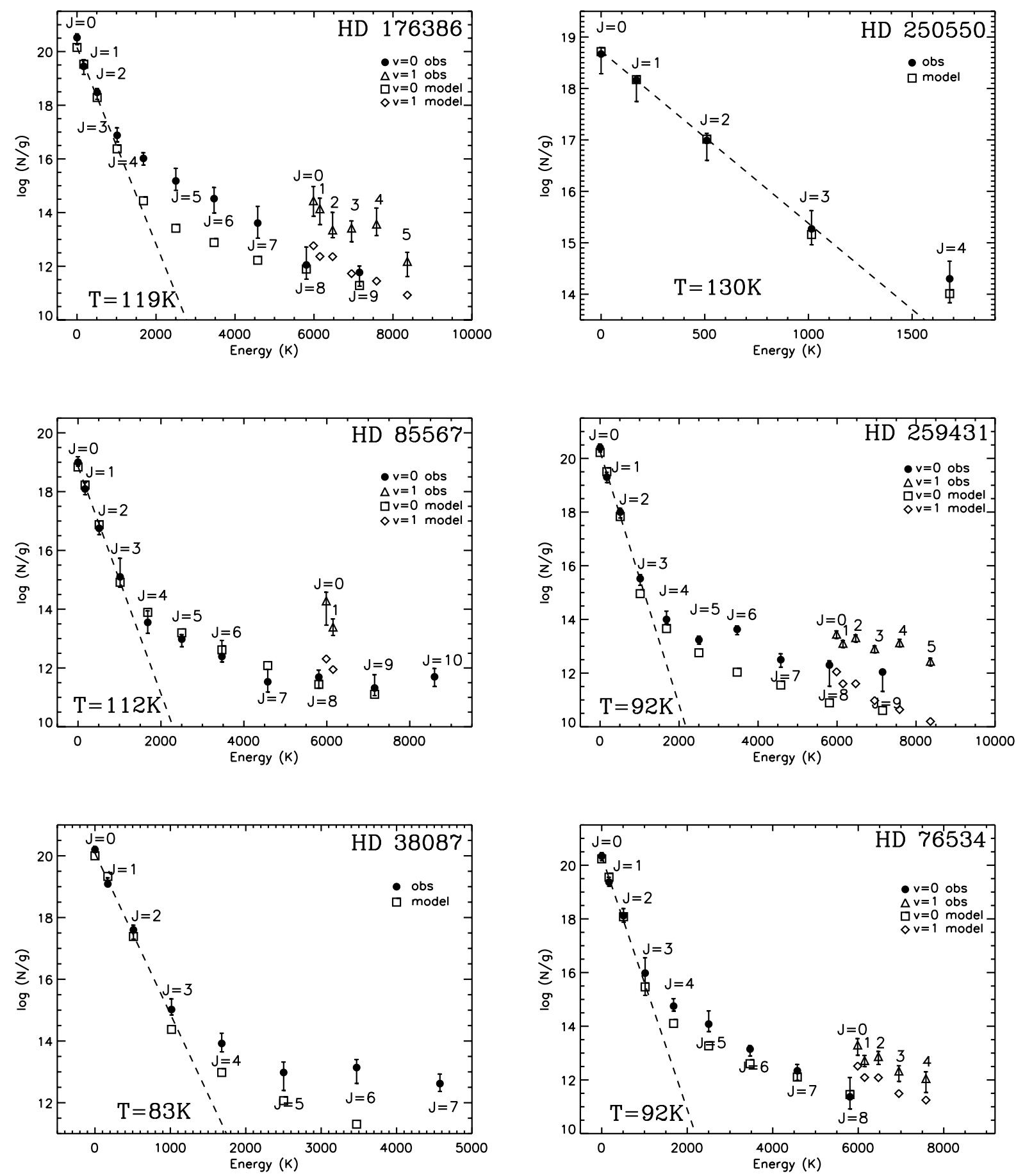

Fig. 6. Best-fitting models obtained with the Meudon PDR Code overplotted on the excitation diagrams derived from the observed column densities (Fig. 5). The general shape of the diagrams are well-reproduced, but the excitation conditions of the higher $J$-levels cannot be reproduced well by the model (see text).

very unlikely. This agrees with the Hillenbrand et al. (1992) analysis of the SED of HD 76534, who stressed the similarity of the very low NIR excess with that of a classical Be star, which is generally thought to be due to free-free emission in an ionized envelope rather than to CS dust (Hamann \& Persson 1992).

\section{Evolutionary trends}

For each group of stars, we explored whether evolutionary trends could be found in our results, especially between the total column densities of $\mathrm{H}_{2}$ in the circumstellar environment of the stars and the ages of the stars.

\subsection{Ae/B9 stars}

As we discussed in Sect. 4, the HAe stars form a very inhomogeneous group. We plotted the column densities of $\mathrm{H}_{2}$ towards the HAe stars versus the inclination angles of their disks in Figs. 13, and 14 the column densities of $\mathrm{H}_{2}$ versus the ages of the HAes of our sample are presented. Both Figs. 13 and 14 are consistent with CS material that is not in disks. They are neither consistent 

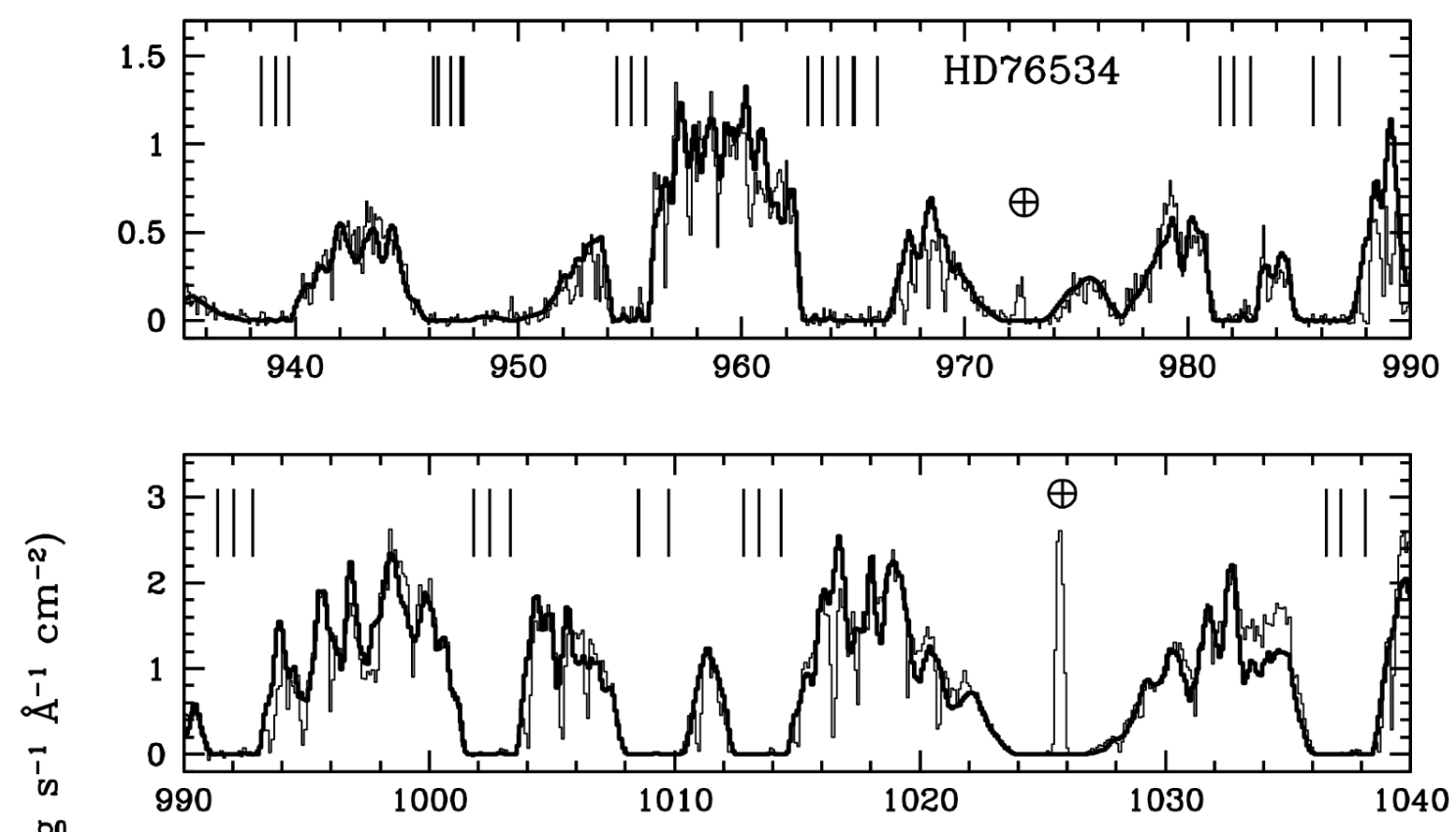

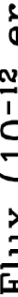
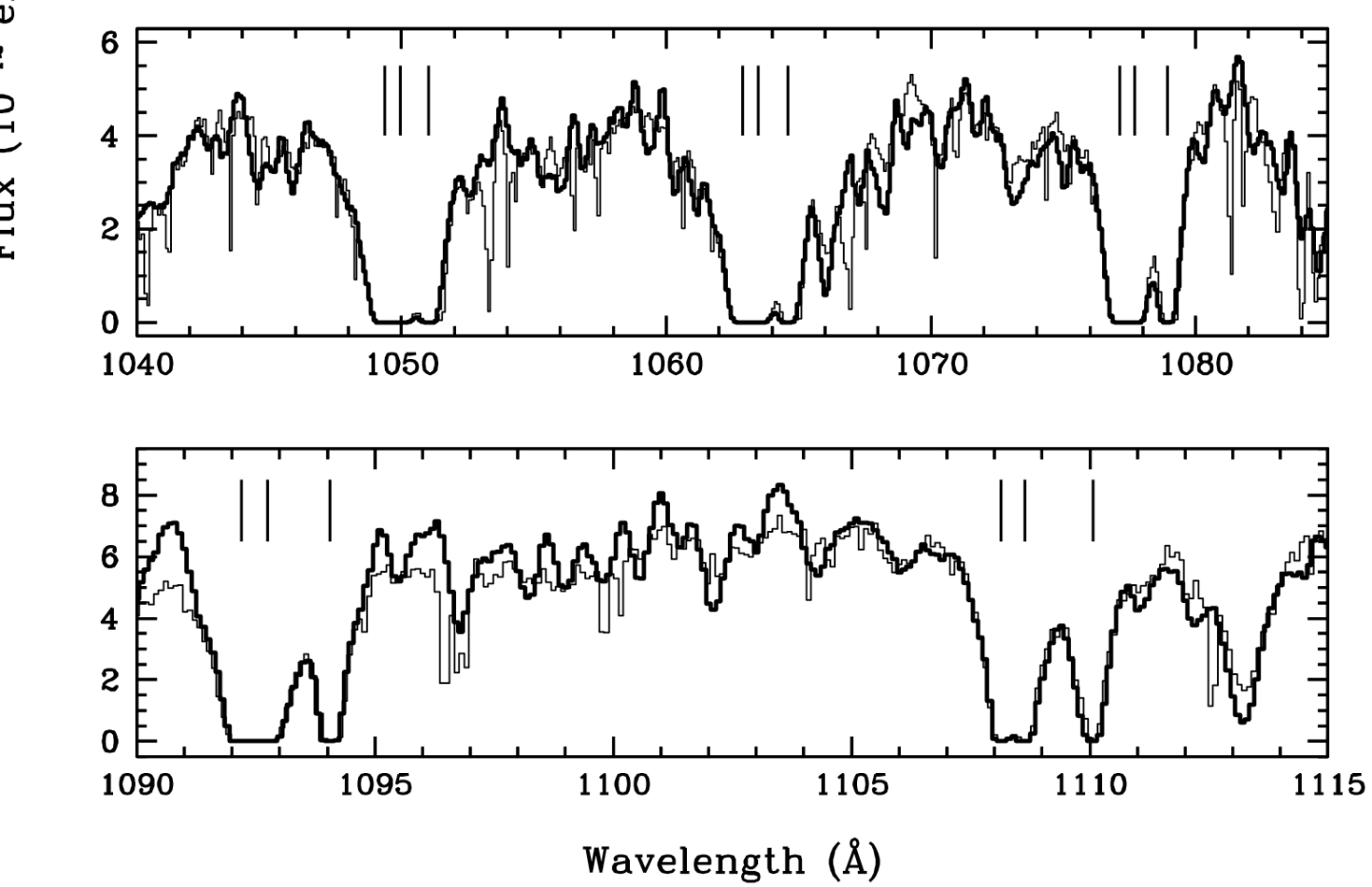

Fig. 7. Overplot of the FUSE spectrum of HD 76534 (thin line) and the synthetic spectrum obtained by combining our photospheric model with the results of the Meudon PDR Code (thick line). This plot only presents the portions of the spectrum where the stronger absorption lines of $\mathrm{H}_{2}(v=0, J=0,1,2)$ are observed. These principal prominent absorption troughs caused by molecular hydrogen are indicated throughout the spectrum. Airglow lines are indicated by $\oplus$ symbols.

with non-associated interstellar clouds nor explained by absorption in the disk material.

On the other hand, when excluding $\beta$ Pictoris and HD 135344, we find that the amount of $\mathrm{H}_{2}$ is higher for the younger stars than for the older ones (Fig. 14). Such a correlation is very unexpected if we observe non-CS media. However, our results do not allow us to rule out CS envelopes around these stars. Indeed, for some of these stars, the presence of both a disk and an envelope has been demonstrated (e.g. Grady et al. 2001). This would confirm the canonical view of star formation, which predicts that the envelope becomes progressively less important for obscuring the star and for dominating the system mass with increasing age. The envelope dominates the mass of the system in the early stages of stellar formation, but when the star is close to the ZAMS, the envelope is expected to have dissipated because of the action of stellar winds prior to central clearing of the disk (see reviews by Calvet et al. 2000; Mundy et al. 2000).

The correlation between the ages of the stars and the total amount of $\mathrm{H}_{2}$ needs to be confirmed. For most stars, we only estimated upper limits on the column densities of $\mathrm{H}_{2}$, and there are large uncertainties on the ages of some of them. Only precise 


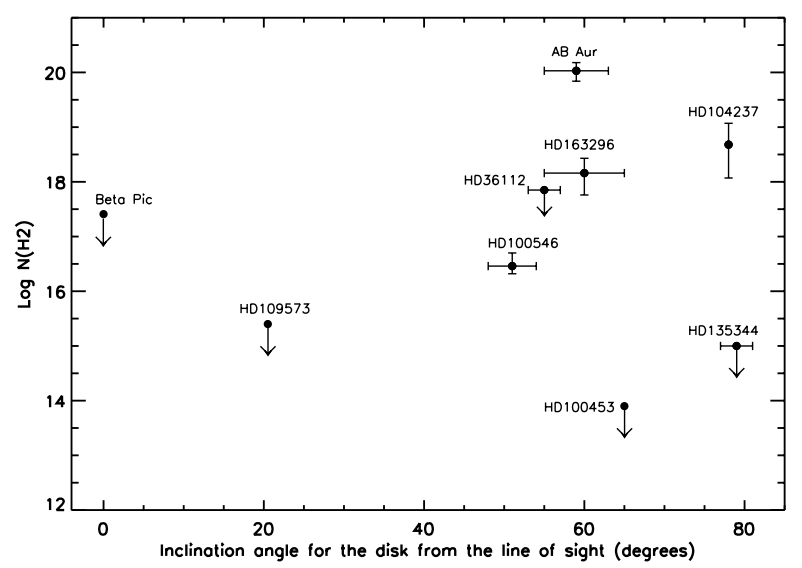

Fig. 13. Total $\mathrm{H}_{2}$ column densities as a function of the inclination angles away from edge-on of the CS disks given in Table 3.

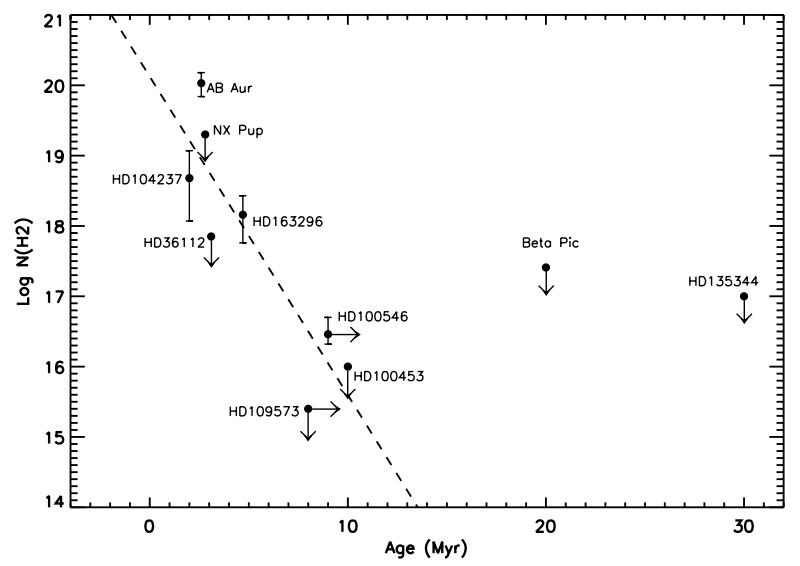

Fig. 14. Total $\mathrm{H}_{2}$ column densities as a function of the ages of those stars known to possess CS disks. Except for $\beta$ Pictoris and HD 135344 , for which we cannot conclude because we only have upper limits, the amount of $\mathrm{H}_{2}$ is greater towards the youngest stars. The correlation between the age and the amount of gas is shown by the dashed line.

measurements of the amount of $\mathrm{H}_{2}$ and the ages of the stars could help to confirm or deny the suspected correlation.

\subsection{Be stars}

Our analysis of the FUSE spectra of the Herbig Be stars shows that this set of stars presents similar $\mathrm{H}_{2}$ physical properties, favoring an interpretation in terms of more or less spherical media around these stars, very likely related to the original clouds in which the stars formed. We find, however, no correlation between the total column densities of $\mathrm{H}_{2}$ and the age of the stars (Fig. 15), as one would expect from rapid dissipation of the original envelope. This lack of correlation could be due to different phenomena: (i) large contamination by the surrounding nebula and thus the presence of different unresolved components along the line of sight; or (ii) uncertainty about the ages of the stars. Herbig Be stars are more massive and more luminous than Ae stars, and the time-scale to reach the ZAMS is very short, making their ages very difficult to estimate.

\section{Summary and conclusions}

We have distinguished two groups of stars: the stars known to possess CS disks (Ae/B9 stars) and the stars with no evidence of

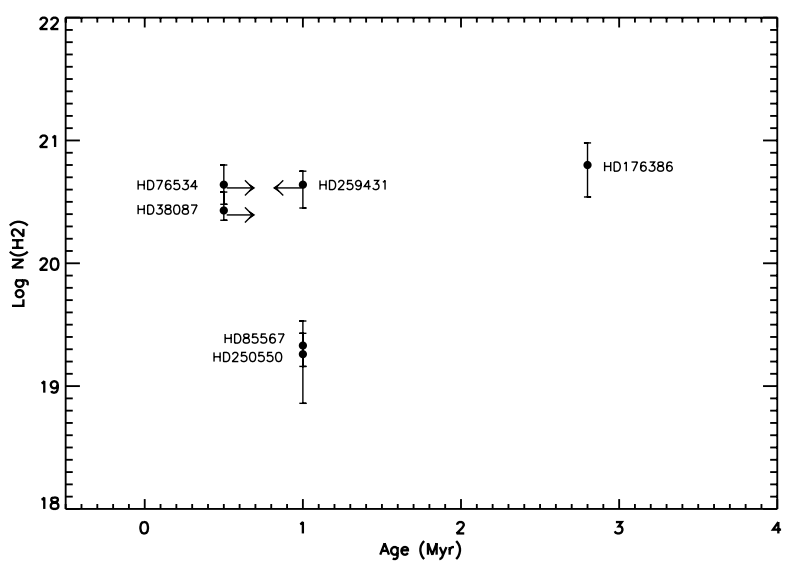

Fig. 15. The total $\mathrm{H}_{2}$ column densities as a function of the ages of the Be stars of our sample, which present no evidence of CS disks.

disks (Be stars). We interpreted the different excitation characteristics of $\mathrm{H}_{2}$ as direct probes of the origin of the observed gas: IS, CS disks, or CS envelopes.

For most of the Herbig Ae/B9 stars, the inclination angles of the CS disks from the lines of sight are quite high and not favorable to probe the disks using absorption spectroscopy. Beta Pictoris and the transitional object HD 109573 are exceptions. In the case of $\beta$ Pictoris we confirm the non-detection of $\mathrm{H}_{2}$ in its FUSE spectrum given in Lecavelier des Etangs et al. (2001). We also did not detect $\mathrm{H}_{2}$ in the spectrum of HD 109573. These nondetections are consistent with the evolved status of these systems and clearing of CS molecular gas from their disks.

AB Aurigae is clearly not edge-on, while $\mathrm{H}_{2}$ is clearly detected, but the cool component of the gas must be located at such a large distance from the star that, even with a relatively large inclination away from edge-on, some disk gas in the very outer regions of the disk may still be in the line of sight.

In the sources HD 36112, HD 135344, and HD 100453, no $\mathrm{H}_{2}$ is observed. This is consistent with the high inclination angles of their disks. The non-detections of gas imply that there are no detectable remnant CS envelopes.

When molecular hydrogen gas is observed in the FUSE spectra of the Herbig Ae/B9 stars of our sample, our analysis shows several kinds of excitation, implying different origins and different physical processes.

In one case, namely HD 141569, the origin of the detected gas is clearly interstellar. For AB Aur there is also some evidence of the interstellar origin on the gas, but we lack the spectral resolution that could allow us to probe the velocity distribution along the line of sight and definitively decide between a CS or IS origin for the gas. In spite of its relatively high inclination away from edge-on, it is not geometrically impossible for the gas to originate in the upper layers of the outer disk regions at about $800 \mathrm{AU}$, but the excitation conditions of $\mathrm{H}_{2}$ towards $\mathrm{AB}$ Aur are fairly typical of what is generally observed in the diffuse interstellar medium. However, we showed that the excitation diagram could not be reproduced well by a single gaseous component along the line of sight. The large line width value favors the presence of two components along the line of sight, which are not resolved in our spectrum. In that case, one of these components could correspond to the remnant of the molecular cloud in which the star formed, as suggested by Roberge et al. (2001). The other component should be hotter and probably closer to the star to explain the excitation of the high $\mathrm{J}$-levels of $\mathrm{H}_{2}$. 
For HD 100546, HD 163296, and HD 104237, we observed excited and probably warm/hot, circumstellar $\mathrm{H}_{2}$ that has excitation conditions clearly different from those observed in the diffuse interstellar medium. Such excitation conditions for $\mathrm{H}_{2}$ give evidence of collisionally excited media close to the stars. The high values of the $b$ parameters support this interpretation. In addition, the measured radial velocities favor a CS origin for the $\mathrm{H}_{2}$. However, assuming that the gas and dust are coupled, the lines of sight towards these three stars do not pass through their disks, and thus the $\mathrm{H}_{2}$ we observed is not located in the disks. This raises questions about the origin of the detected gas. One interesting possibility could be that the $\mathrm{H}_{2}$ gas is an FUV-driven photoevaporative wind from the outer parts of the disk, but we are still missing clues needed for a firm conclusion. The Meudon PDR code did not allow us to reproduce the excitation of the observed $\mathrm{H}_{2}$ gas. This implies that peculiar excitation processes such as shocks or X-rays, not taken into account in the Meudon PDR Code, may play a role in the environment of these stars.

The excitation conditions of circumstellar $\mathrm{H}_{2}$ around the stars of our second group (Be stars) are clearly different from those of the first group. We found similar excitation conditions for the $\mathrm{H}_{2}$ from one star to the next. They present similarities to the conditions found in the IS medium. Our analysis favors an interpretation in terms of spherically symmetric media, not affected by inclination effects. The excitation diagrams are reproduced nicely by PDR models, at least for the cold component that includes more than $90 \%$ of the gas. On the other hand, these models do not simultaneously reproduce the excitation conditions of the higher $J$-levels of $\mathrm{H}_{2}$. We very likely observe complex environments, such as large CS envelopes, remnants of the original clouds in which the stars formed. However, other excitation processes, in addition to those taken into account in the Meudon PDR Code, are probably necessary to fully explain the observed excitation diagrams.

Our FUV spectral analysis reinforces the differences between the two subclasses of stars (Ae and Be) already highlighted by different authors (e.g. Natta et al. 2000). It also points to the need for complementary observations to better understand the physical conditions of formation and excitation of $\mathrm{H}_{2}$ and the origin of the gas. We suggest two avenues for bringing significant insight into these issues. The first will be to observe spectral lines of the $\mathrm{CH}$ and $\mathrm{CH}^{+}$molecules in the optical range at high spectral resolution. These molecules are linked to the formation and excitation of $\mathrm{H}_{2}$ (Federman 1982; Mattila 1986; Somerville \& Smith 1989). The formation of $\mathrm{CH}$ is predicted to be controlled by gas-phase reactions with $\mathrm{H}_{2}$. The $\mathrm{CH}$ is thus a good tracer of $\mathrm{H}_{2}$ and their abundances are generally strongly correlated. In the presence of a shock, the formation of the $\mathrm{CH}^{+}$molecule through the chemical reaction $\mathrm{C}^{+}+\mathrm{H}_{2}$ needs a temperature about of $4500 \mathrm{~K}$ to occur. Thus, the $\mathrm{CH}^{+}$molecule is a probe of hot and excited media, which could be interpreted for our targets as material close to the star, and this would allow us to better constrain the excitation of $\mathrm{H}_{2}$. The observation of $\mathrm{CH}$ and $\mathrm{CH}^{+}$, if present, would provide direct evidence of warm/hot CS gas close to the star. Combined with the $\mathrm{H}_{2}$ data, this will allow us to explain the chemical mechanisms of formation/destruction and excitation of the different molecules in the CS environment of HAeBes and to better evaluate the velocity dispersion along the line of sight (possibly due to turbulence).

The second avenue would be to observe pure rotational and ro-vibrational transitions of $\mathrm{H}_{2}$ in the infrared wavelength range. This would give access to the spatial distribution and mass of the observed gas and would help constrain the excitation mechanisms. Such infrared observations would also provide information about the circumstellar dust, including PAHs. Nextgeneration instruments like VLT/VISIR could provide these kinds of observations (Martin-Zaïdi et al. 2007).

All these data would give us the opportunity to obtain a global picture of both the structure and the evolution of the CS environment of HAeBes.

Acknowledgements. This research is based on observations made with the NASA-CNES-CSA Far Ultraviolet Spectroscopic Explorer. FUSE is operated for NASA by the Johns Hopkins University under NASA contract NAS5-32985. This work was done using the profile fitting procedure Owens.f developed by M. Lemoine and the FUSE French Team. C. M.-Z. warmly thanks David Hollenbach for fruitful discussions about the possible sources of hot excited $\mathrm{H}_{2}$. We thank D. Ehrenreich, B. Godard at JHU, and J.-C. Meunier at the LAM for their help in reprocessing the data.

\section{References}

Abgrall, H., Roueff, E., Launay, F., Roncin, J. Y., \& Subtil, J. L. 1993a, A\&AS, 101,273

Abgrall, H., Roueff, E., Launay, F., Roncin, J. Y., \& Subtil, J. L. 1993b, A\&AS, 101,323

Abgrall, H., Roueff, E., \& Drira, I. 2000, A\&AS, 141, 297

Acke, B., \& Waelkens, C. 2004, A\&A, 427, 1009

Augereau, J. C., \& Papaloizou, J. C. B. 2004, A\&A, 414, 1153

Augereau, J. C., Lagrange, A. M., Mouillet, D., \& Ménard, F. 1999, A\&A, 350, L51

Augereau, J. C., Lagrange, A. M., Mouillet, D., \& Ménard, F. 2001, A\&A, 365, 78

Barrado y Navascués, D., Stauffer, J. R., Song, I., \& Caillault, J.-P. 1999, ApJ, 520, L123

Bastien, P., \& Menard, F. 1990, ApJ, 364, 232

Bertout, C., Robichon, N., \& Arenou, F. 1999, A\&A, 352, 574

Beskrovnaya, N. G., Pogodin, M. A., Miroshnichenko, A. S., et al. 1999, A\&A, 343, 163

Bitner, M. A., Richter, M. J., Lacy, J. H., et al. 2007, ApJ, 661, L69

Bohlin, R. C., Savage, B. D., \& Drake, J. F. 1978, ApJ, 224, 132

Böhm, T., \& Catala, C. 1993, A\&AS, 101, 629

Böhm, T., \& Catala, C. 1995, A\&A, 301, 155

Boissé, P., Le Petit, F., Rollinde, E., et al. 2005, A\&A, 429, 509

Bouret, J.-C., \& Catala, C. 1998, A\&A, 340, 163

Bouret, J.-C., Deleuil, M., Lanz, T., et al. 2002, A\&A, 390, 1049

Bouret, J.-C., Martin, C., Deleuil, M., Simon, T., \& Catala, C. 2003, A\&A, 410, 175

Bouwman, J., de Koter, A., Dominik, C., \& Waters, L. B. F. M. 2003, A\&A, 401, 577

Browning, M. K., Tumlinson, J., \& Shull, J. M. 2003, ApJ, 582, 810

Burgh, E. B., McCandliss, S. R., \& Feldman, P. D. 2002, ApJ, 575, 240

Calvet, N., Hartmann, L., \& Strom, S. E. 2000, Protostars and Planets IV, 377

Chen, C. H., \& Kamp, I. 2004, ApJ, 602, 985

Chieze, J.-P., Pineau des Forets, G., \& Flower, D. R. 1998, MNRAS, 295, 672

Cidale, L., Zorec, J., \& Tringaniello, L. 2001, A\&A, 368, 160

Corcoran, M., \& Ray, T. P. 1997, A\&A, 321, 189

Crifo, F., Vidal-Madjar, A., Lallement, R., Ferlet, R., \& Gerbaldi, M. 1997, A\&A, 320, L29

Deleuil, M., Lecavelier des Etangs, A., Bouret, J.-C., et al. 2004, A\&A, 418, 577

Deleuil, M., Bouret, J.-C., Catala, C., et al. 2005, A\&A, 429, 247

Deleuil, M., Bouret, J. C., Lecavelier Des Etangs, A., Roberge, A., \& Feldman, P. 2006, in Astrophysics in the Far Ultraviolet: Five Years of Discovery with FUSE, ed. G. Sonneborn, H. W. Moos, \& B.-G. Andersson, ASP Conf. Ser., 348, 297

Dent, W. R. F., Greaves, J. S., \& Coulson, I. M. 2005, MNRAS, 359, 663

Dixon, W. V., Sahnow, D. J., Barrett, P. E., et al. 2007, PASP, 119, 527

Dominik, C., Dullemond, C. P., Waters, L. B. F. M., \& Walch, S. 2003, A\&A, 398,607

Donati, J.-F., Semel, M., Carter, B. D., Rees, D. E., \& Collier Cameron, A. 1997, MNRAS, 291, 658

Draine, B. T. 1978, ApJS, 36, 595

Dunkin, S. K., Barlow, M. J., \& Ryan, S. G. 1997, MNRAS, 286, 604

Eisner, J. A., Lane, B. F., Akeson, R. L., Hillenbrand, L. A., \& Sargent, A. I. 2003, ApJ, 588, 360

Eisner, J. A., Lane, B. F., Hillenbrand, L. A., Akeson, R. L., \& Sargent, A. I. 2004, ApJ, 613, 1049

Falgarone, E., \& Puget, J.-L. 1995, A\&A, 293, 840 
Federman, S. R. 1982, ApJ, 257, 125

Finkenzeller, U., \& Jankovics, I. 1984, A\&AS, 57, 285

Gerbaldi, M., Faraggiana, R., Burnage, R., et al. 1999, A\&AS, 137, 273

Grady, C. A., Devine, D., Woodgate, B., et al. 2000, ApJ, 544, 895

Grady, C. A., Polomski, E. F., Henning, T., et al. 2001, AJ, 122, 3396

Grady, C., Woodgate, B., Torres, C. A. O., et al. 2004, ApJ, 608, 809

Gredel, R., Pineau des Forêts, G., \& Federman, S. R. 2002, A\&A, 389, 993

Gry, C., Boulanger, F., Nehmé, C., et al. 2002, A\&A, 391, 675

Hébrard, G., Lemoine, M., Vidal-Madjar, A., et al. 2002, ApJS, 140, 103

Hamann, F., \& Persson, S. E. 1992, ApJ, 394, 628

Herczeg, G. J., Linsky, J. L., Brown, A., Harper, G. M., \& Wilkinson, E. 2003

in The Future of Cool-Star Astrophysics: 12th Cambridge Workshop on Cool

Stars, Stellar Systems, and the Sun (2001 July 30-August 3), ed. A. Brown,

G. M. Harper, \& T. R. Ayres (University of Colorado), 717

Hernández, J., Calvet, N., Briceño, C., Hartmann, L., \& Berlind, P. 2004, AJ, 127,1682

Hillenbrand, L. A., Strom, S. E., Vrba, F. J., \& Keene, J. 1992, ApJ, 397, 613

Hollenbach, D., Johnstone, D., Lizano, S., \& Shu, F. 1994, ApJ, 428, 654

Joulain, K., Falgarone, E., Des Forets, G. P., \& Flower, D. 1998, A\&A, 340, 241

Jura, M. 1991, ApJ, 383, L79

Juvela, M., Mattila, K., Lehtinen, K., et al. 2002, A\&A, 382, 583

Lada, C. J. 1985, ARA\&A, 23, 267

Lada, C. J., \& Adams, F. C. 1992, ApJ, 393, 278

Lagage, P.-O., Doucet, C., Pantin, E., et al. 2006, Science, 314, 621

Le Bourlot, J., Pineau Des Forets, G., Roueff, E., \& Flower, D. R. 1993, A\&A, 267,233

Le Bourlot, J., Pineau des Forêts, G., \& Flower, D. R. 1999, MNRAS, 305, 802

Le Petit, F., Nehmé, C., Le Bourlot, J., \& Roueff, E. 2006, ApJS, 164, 506

Lecavelier des Etangs, A., Vidal-Madjar, A., Roberge, A., et al. 2001, Nature, 412, 706

Lecavelier des Etangs, A., Deleuil, M., Vidal-Madjar, A., et al. 2003, A\&A, 407, 935

Lehner, N., Jenkins, E. B., Gry, C., et al. 2003, ApJ, 595, 858

Leinert, C., Haas, M., Ábrahám, P., \& Richichi, A. 2001, A\&A, 375, 927

Lemoine, M., Vidal-Madjar, A., Hébrard, G., et al. 2002, ApJS, 140, 67

Malfait, K., Bogaert, E., \& Waelkens, C. 1998, A\&A, 331, 211

Mannings, V., \& Sargent, A. I. 1997, ApJ, 490, 792

Mannings, V., \& Sargent, A. I. 2000, ApJ, 529, 391

Martin, C., Bouret, J.-C., Deleuil, M., Simon, T., \& Catala, C. 2004, A\&A, 416, L5

Martin-Zaïdi, C., Deleuil, M., Simon, T., et al. 2005, A\&A, 440, 921

Martin-Zaïdi, C., Lagage, P.-O., Pantin, E., \& Habart, E. 2007, ApJ, 666, L117

Mathis, J. S., Rumpl, W., \& Nordsieck, K. H. 1977, ApJ, 217, 425

Mattila, K. 1986, A\&A, 160, 157

Meeus, G., Waters, L. B. F. M., Bouwman, J., et al. 2001, A\&A, 365, 476
Meeus, G., Bouwman, J., Dominik, C., Waters, L. B. F. M., \& de Koter, A. 2002, A\&A, 392, 1039

Millan-Gabet, R., Schloerb, F. P., \& Traub, W. A. 2001, ApJ, 546, 358

Miroshnichenko, A. S., Levato, H., Bjorkman, K. S., \& Grosso, M. 2001, A\&A, 371,600

Mora, A., Merín, B., Solano, E., et al. 2001, A\&A, 378, 116

Mundy, L. G., Looney, L. W., \& Welch, W. J. 2000, Protostars and Planets IV, 355

Natta, A., Grinin, V., \& Mannings, V. 2000, Protostars and Planets IV, 559

Palla, F., \& Stahler, S. W. 1993, ApJ, 418, 414

Pantin, E., Waelkens, C., \& Lagage, P. O. 2000, A\&A, 361, L9

Pantin, E., Bouwman, J., \& Lagage, P. O. 2005, A\&A, 437, 525

Polomski, E. F., Telesco, C. M., Piña, R., \& Schulz, B. 2002, AJ, 124, 2207

Proust, D., Ochsenbein, F., \& Pettersen, B. R. 1981, A\&AS, 44, 179

Redfield, S., \& Linsky, J. L. 2004, ApJ, 613, 1004

Rice, W. K. M., Wood, K., Armitage, P. J., Whitney, B. A., \& Bjorkman, J. E. 2003, MNRAS, 342, 79

Richter, M. J., Jaffe, D. T., Blake, G. A., \& Lacy, J. H. 2002, ApJ, 572, L161

Roberge, A., Lecavelier des Etangs, A., Grady, C. A., et al. 2001, ApJ, 551, L97

Roberge, A., Feldman, P. D., Weinberger, A. J., Deleuil, M., \& Bouret, J.-C. 2006, Nature, 441, 724

Royer, F., Grenier, S., Baylac, M.-O., Gómez, A. E., \& Zorec, J. 2002, A\&A, 393, 897

Sako, S., Yamashita, T., Kataza, H., et al. 2005, ApJ, 620, 347

Semenov, D., Pavlyuchenkov, Y., Schreyer, K., et al. 2005, ApJ, 621, 853

Setiawan, J., Henning, T., Launhardt, R., et al. 2008, Nature, 451, 38

Shull, J. M., \& Beckwith, S. 1982, ARA\&A, 20, 163

Siebenmorgen, R., Prusti, T., Natta, A., \& Müller, T. G. 2000, A\&A, 361, 258

Smith, B. A., \& Terrile, R. J. 1984, Science, 226, 1421

Snow, T. P., \& Witt, A. 1989, ApJ, 342, 295

Snow, T. P., \& McCall, B. J. 2006, ARA\&A, 44, 367

Somerville, W. B., \& Smith, C. A. 1989, MNRAS, 238, 559

Spitzer, L. J., \& Cochran, W. D. 1973, ApJ, 186, L23

Stapelfeldt, K. R., Burrows, C. J., Krist, J. E., et al. 1998, ApJ, 508, 736

Telesco, C. M., Fisher, R. S., Piña, R. K., et al. 2000, ApJ, 530, 329

Testi, L., Palla, F., \& Natta, A. 1998, A\&AS, 133, 81

Thé, P. S., de Winter, D., \& Perez, M. R. 1994, A\&AS, 104, 315

Thi, W. F., van Dishoeck, E. F., Blake, G. A., et al. 2001, ApJ, 561, 1074

Torres, G., Guenther, E. W., Marschall, L. A., et al. 2003, AJ, 125, 825

Valenti, J. A., Johns-Krull, C. M., \& Linsky, J. L. 2000, ApJS, 129, 399

van den Ancker, M. E., de Winter, D., \& Tjin A Djie, H. R. E. 1998, A\&A, 330, 145

Vaz, L. P. R., Andersen, J., Casey, B. W., et al. 1998, A\&AS, 130, 245

Wahhaj, Z., Koerner, D. W., Backman, D. E., et al. 2005, ApJ, 618, 385

Weinberger, A. J., Becklin, E. E., Schneider, G., et al. 1999, ApJ, 525, L53 
C. Martin-Zaïdi et al.: $\mathrm{H}_{2}$ in the CS environment of HAeBes, Online Material $p 1$

Table 4. Column densities of the different energy levels of $\mathrm{H}_{2}$ when clearly observed in the FUSE spectra of the stars. " $g$ " is the statistical weight of each level.

\begin{tabular}{|c|c|c|c|c|c|c|c|c|c|c|c|c|c|c|}
\hline$v$ & $J$ & $g$ & $\begin{array}{c}\text { Energy } \\
\text { level }(\mathrm{K})\end{array}$ & $\begin{array}{c}\text { HD } 104237 \\
\log N \\
\left(\mathrm{~cm}^{-2}\right)\end{array}$ & $\begin{array}{c}\text { HD } 163296 \\
\log N \\
\left(\mathrm{~cm}^{-2}\right)\end{array}$ & $\begin{array}{l}\text { AB Aur } \\
\log N \\
\left(\mathrm{~cm}^{-2}\right)\end{array}$ & $\begin{array}{c}\text { HD } 141569 \\
\log N \\
\left(\mathrm{~cm}^{-2}\right)\end{array}$ & $\begin{array}{c}\text { HD } 100546 \\
\log N \\
\left(\mathrm{~cm}^{-2}\right)\end{array}$ & $\begin{array}{c}\text { HD } 176386 \\
\log N \\
\left(\mathrm{~cm}^{-2}\right)\end{array}$ & $\begin{array}{c}\text { HD } 250550 \\
\log N \\
\left(\mathrm{~cm}^{-2}\right)\end{array}$ & $\begin{array}{c}\text { HD } 85567 \\
\log N \\
\left(\mathrm{~cm}^{-2}\right)\end{array}$ & $\begin{array}{c}\text { HD } 259431 \\
\log N \\
\left(\mathrm{~cm}^{-2}\right)\end{array}$ & $\begin{array}{c}\operatorname{HD} 38087 \\
\log N \\
\left(\mathrm{~cm}^{-2}\right)\end{array}$ & $\begin{array}{c}\text { HD } 76534 \\
\log N \\
\left(\mathrm{~cm}^{-2}\right)\end{array}$ \\
\hline 0 & 0 & 1 & 0.00 & $17.85_{-0.73}^{+0.14}$ & $17.10_{-0.70}^{+0.40}$ & $19.85_{-0.15}^{+0.13}$ & $20.08_{-0.09}^{+0.21}$ & $15.16_{-0.26}^{+0.38}$ & $20.52_{-0.26}^{+0.14}$ & $18.67_{-0.14}^{+0.37}$ & $19.00_{-0.14}^{+0.19}$ & $20.40_{-0.16}^{+0.13}$ & $20.20_{-0.08}^{+0.10}$ & $20.34_{-0.171}^{+0.131}$ \\
\hline 0 & 1 & 9 & 170.49 & $18.32_{-0.56}^{+0.39}$ & $17.90_{-0.30}^{+0.20}$ & $19.56_{-0.27}^{+0.13}$ & $19.96_{-0.48}^{+0.18}$ & $16.00_{-0.19}^{+0.28}$ & $20.40_{-0.30}^{+0.20}$ & $19.11_{-0.07}^{+0.07}$ & $19.04_{-0.19}^{+0.21}$ & $20.25_{-0.20}^{+0.10}$ & $20.05_{-0.08}^{+0.08}$ & $20.32_{-0.154}^{+0.181}$ \\
\hline 0 & 2 & 5 & 509.897 & $17.99_{-0.63}^{+0.30}$ & $17.40_{-100}^{+0.40}$ & $16.60_{-0.37}^{+0.27}$ & $\begin{array}{r}-0.48 \\
16.20_{-0.69}^{+0.69}\end{array}$ & $15.58_{-028}^{+0.19}$ & $19.18_{-0.06}^{-0.30}$ & $17.69_{-0.039}^{-0.41}$ & $17.45^{-0.25}$ & $18.70^{-0.14}$ & $18.30^{-0.08}+15$ & $18.82^{-0.263}$ \\
\hline 0 & 3 & 21 & 1015.069 & $17.98_{-0.61}^{+0.63}$ & $17.40_{-0.30}^{+0.00}$ & $16.65_{-0.20}^{+0.44}$ & $16.04_{-0.55}^{+0.38}$ & $15.83_{-0.11}^{+0.21}$ & $18.20_{-0.24}^{-0.26}$ & $16.59_{-0.31}^{-0.39}$ & $16.42^{+0.63}$ & $16.85^{-0.15}$ & $16.34_{-0.34}^{+0.30}$ & $17.30_{-0.530}^{+0.571}$ \\
\hline 0 & 4 & 9 & 1681.281 & $16.19_{-0.59}^{+0.01}$ & $\begin{array}{r}16.40_{-0.80}^{+0.40} \\
16.0\end{array}$ & $15.75_{-0.30}^{+0.20}$ & $15.78_{-0.84}^{-0.37}$ & $15.13_{-0.07}^{+0.11}$ & $16.98_{-0.25}^{-0.24}$ & $15.26_{-0.47}^{-0.31}$ & $\begin{array}{r}-0.35 \\
14.50^{+0.39}\end{array}$ & $14.95^{-0.31}$ & $14.88^{-0.33}$ & $\begin{array}{r}-0.830 \\
15.70^{+0.278}\end{array}$ \\
\hline 0 & 5 & 33 & 2502.234 & $\begin{array}{l}16.70_{-0.42}^{+0.56} \\
\end{array}$ & --0.80 & $15.30_{-0.40}^{+0.50}$ & $--^{-0.84}$ & $15.51_{-0.06}^{+0.07}$ & $16.70_{-0.45}^{-0.25}$ & $-^{-0.4 /}$ & $14.50^{-0.15}$ & $14.75^{-0.16}$ & $14.50^{-0.34}$ & $15.60^{-0.494}$ \\
\hline 0 & 6 & 13 & 3470.052 & $-^{-0.42}$ & - & $13.45_{-0.35}^{-0.40}$ & - & $14.69_{-0.10}^{-0.06}$ & $15.63_{-0.54}^{-0.35}$ & - & $13.50_{-0.19}^{+0.54}$ & $14.74_{-0.24}^{+0.17}$ & $14.25_{-0.51}^{+0.26}$ & $14.26_{-0.260}^{+0.128}$ \\
\hline 0 & 7 & 45 & 4575.286 & - & - & - & - & $14.87_{-0.08}^{+0.10}$ & $15.26_{-0.56}^{+0.54}$ & - & $13.18_{-0.45}^{+0.19}$ & $14.15_{-0.35}^{-0.15}$ & $14.27_{-0.25}^{+0.31}$ & $13.99_{-0.359}^{+0.236}$ \\
\hline 0 & 8 & 17 & 5806.903 & - & - & - & - & $13.89_{-0.25}^{+0.08}$ & $13.28_{-0.53}^{+0.56}$ & - & $\begin{array}{r}-0.35 \\
12.92_{-0.38}^{+0.24}\end{array}$ & $13.53^{-0.23}$ & -0.25 & $12.60^{+0.721}$ \\
\hline 0 & 9 & 57 & 7152.314 & - & - & - & - & $14.31_{-0.08}^{-0.25}$ & $13.53_{-0.54}^{+0.23}$ & - & $13.08_{-0.45}^{+0.38}$ & $13.80^{-1.42}$ & - & -0.451 \\
\hline 0 & 10 & 21 & 8597.343 & - & - & - & - & $13.47_{-0.55}^{+0.01}$ & - & - & $13.02_{-0.28}^{+0.20}$ & - & - & - \\
\hline 1 & 0 & 1 & 5986.847 & - & - & - & - & - & $14.44_{-0.58}^{+0.53}$ & - & $14.28_{-0.82}^{+0.30}$ & $13.45^{+0.12}$ & - & $13.30^{+0.239}$ \\
\hline 1 & 1 & 9 & 6149.431 & - & - & - & - & $13.87_{-0.05}^{+0.05}$ & $15.09_{-059}^{+0.38}$ & - & $14.34_{-0.28}^{+0.82}$ & $14.07^{-0.03}$ & - & $13.68^{-0.380}+190$ \\
\hline 1 & 2 & 5 & 6471.722 & - & - & - & - & $13.45_{-0.11}^{+0.00}$ & $14.05_{-0.28}^{+0.59}$ & - & $-^{-0.28}$ & $14.02^{-0.0 .08}$ & - & $13.57^{-0.191}$ \\
\hline 1 & 3 & 21 & 6950.843 & - & - & - & - & $13.91_{-0.04}^{+0.111}$ & $14.74_{-0.57}^{-0.28}$ & - & - & $14.23^{-0.19}$ & - & $13.65^{-0.195}$ \\
\hline 1 & 4 & 9 & 7583.915 & - & - & - & - & $13.33_{-0.09}^{+0.04}$ & $14.52_{-0.43}^{-0.51}$ & - & - & $14.09^{-0.08}$ & - & $13.00^{-0.386}+0.253$ \\
\hline 1 & 5 & 33 & 8363.744 & - & - & - & - & $13.89_{-0.08}^{+0.09}$ & $13.70_{-0.56}^{+0.34}$ & - & - & $13.96_{-017}^{-0.01}$ & - & - \\
\hline
\end{tabular}
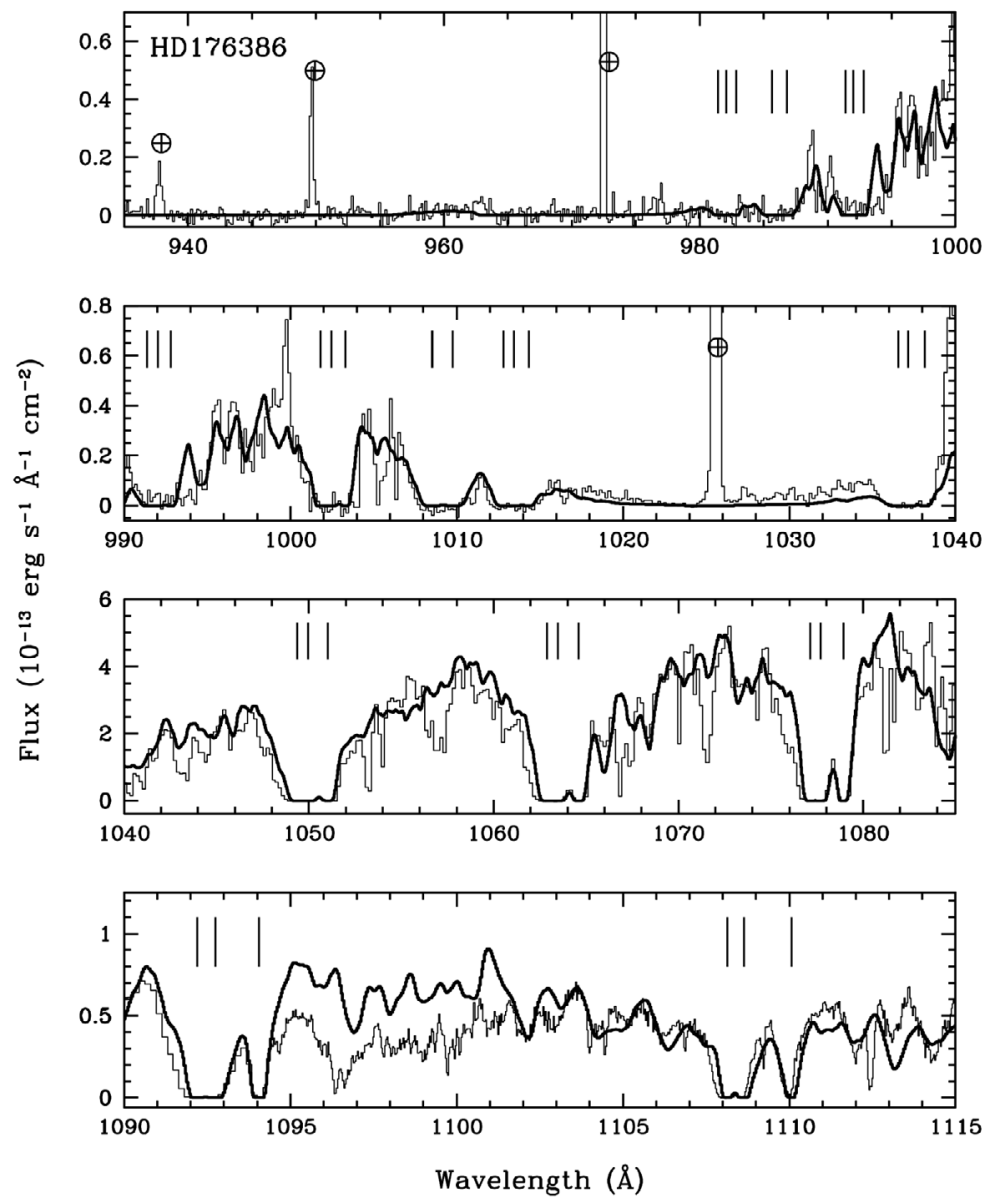

Fig. 8. Overplot of the FUSE spectrum of HD 176386 (thin line) and the synthetic spectrum obtained by combining our photospheric model with the results of the Meudon PDR Code (thick line). This plot only presents the portions of the spectrum where the stronger absorption lines of $\mathrm{H}_{2}(v=0, J=0,1,2)$ are observed. These principal prominent absorption troughs caused by molecular hydrogen are indicated throughout the spectrum. Airglow lines are indicated by $\oplus$ symbols. 
C. Martin-Zaïdi et al.: $\mathrm{H}_{2}$ in the CS environment of HAeBes, Online Material p 2
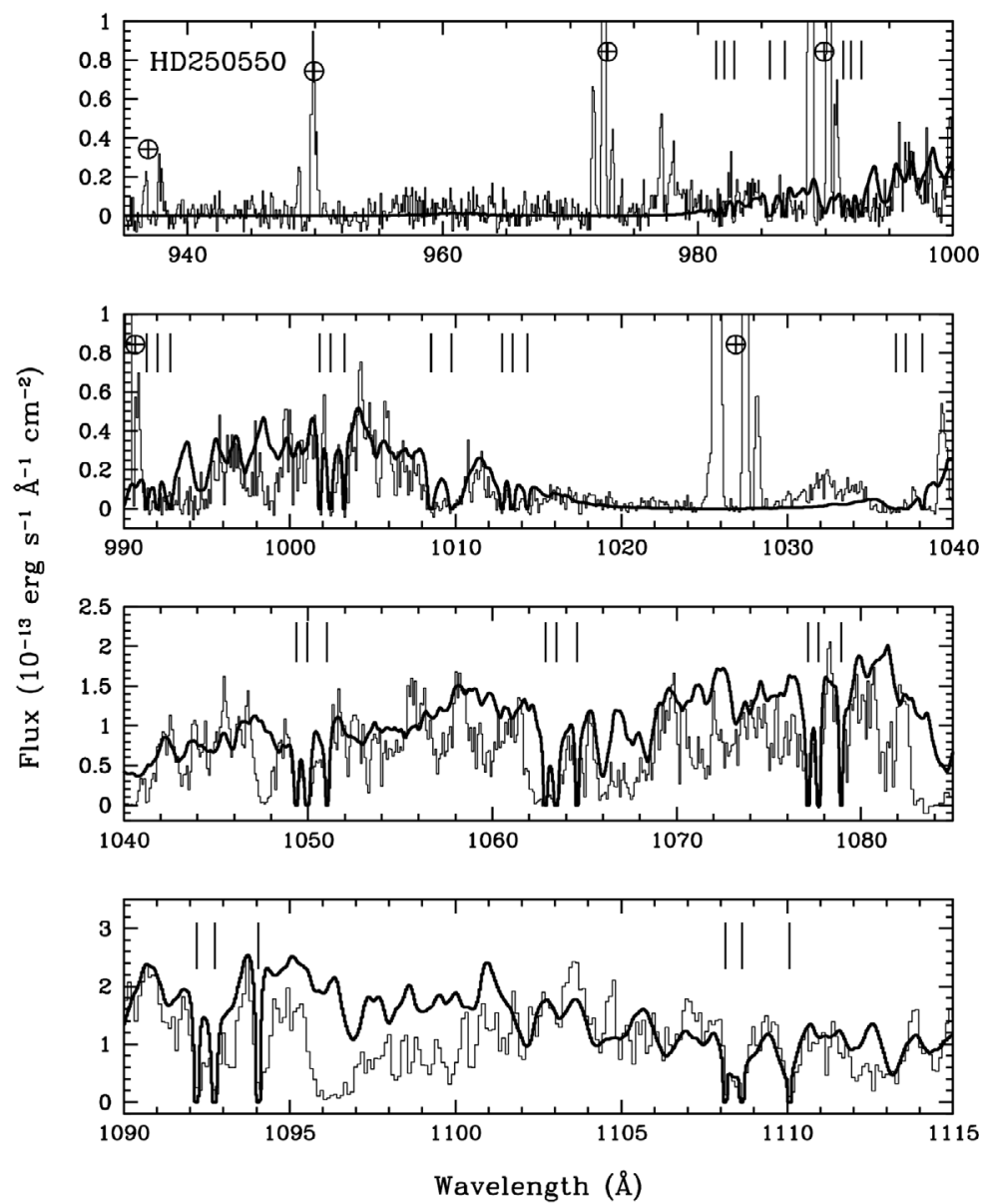

Fig. 9. Overplot of the FUSE spectrum of HD 250550 (thin line) and the synthetic spectrum obtained by combining our photospheric model with the results of the Meudon PDR Code (thick line). Same legend as for Fig. 8. Here, the photospheric model does not fit the data well because of the strong stellar wind lines (for details see Bouret et al. 2003). 
C. Martin-Zaïdi et al.: $\mathrm{H}_{2}$ in the CS environment of HAeBes, Online Material p 3
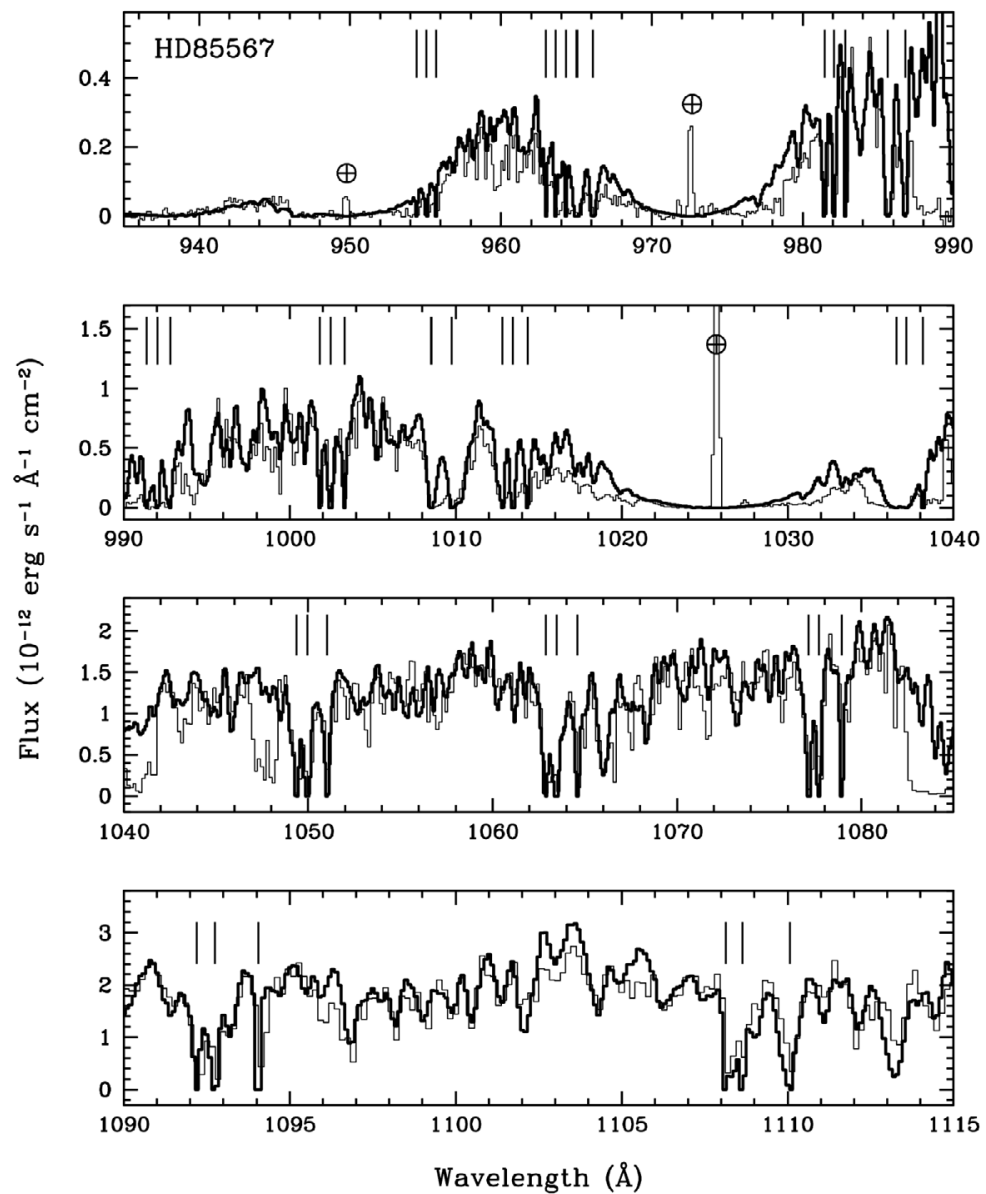

Fig. 10. Overplot of the FUSE spectrum of HD 85567 (thin line) and the synthetic spectrum obtained by combining our photospheric model with the results of the Meudon PDR Code (thick line). Same legend as for Fig. 8. 
C. Martin-Zaïdi et al.: $\mathrm{H}_{2}$ in the CS environment of HAeBes, Online Material p 4
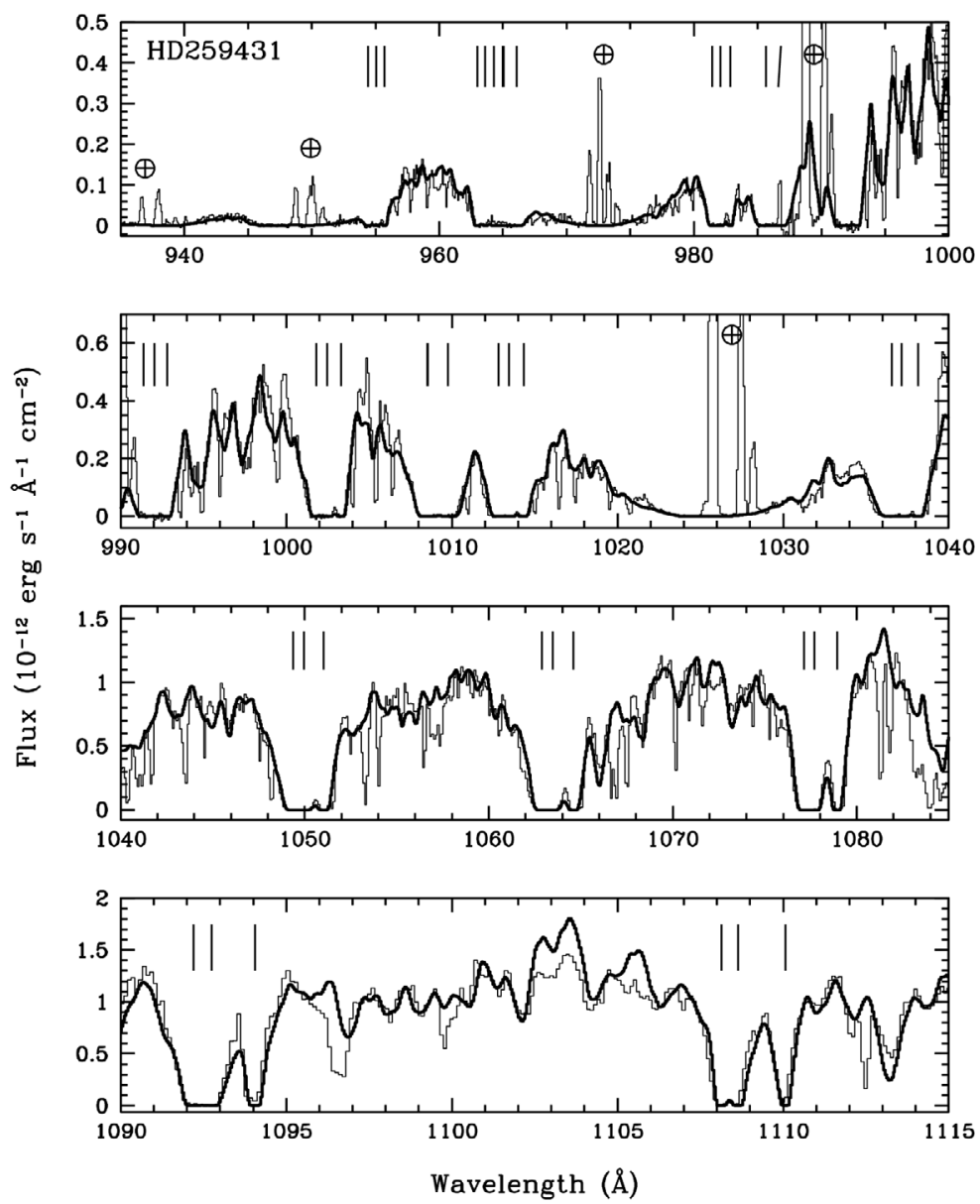

Fig. 11. Overplot of the FUSE spectrum of HD 259431 (thin line) and the synthetic spectrum obtained by combining our photospheric model with the results of the Meudon PDR Code (thick line). Same legend as for Fig. 8. 
C. Martin-Zaïdi et al.: $\mathrm{H}_{2}$ in the CS environment of HAeBes, Online Material p 5
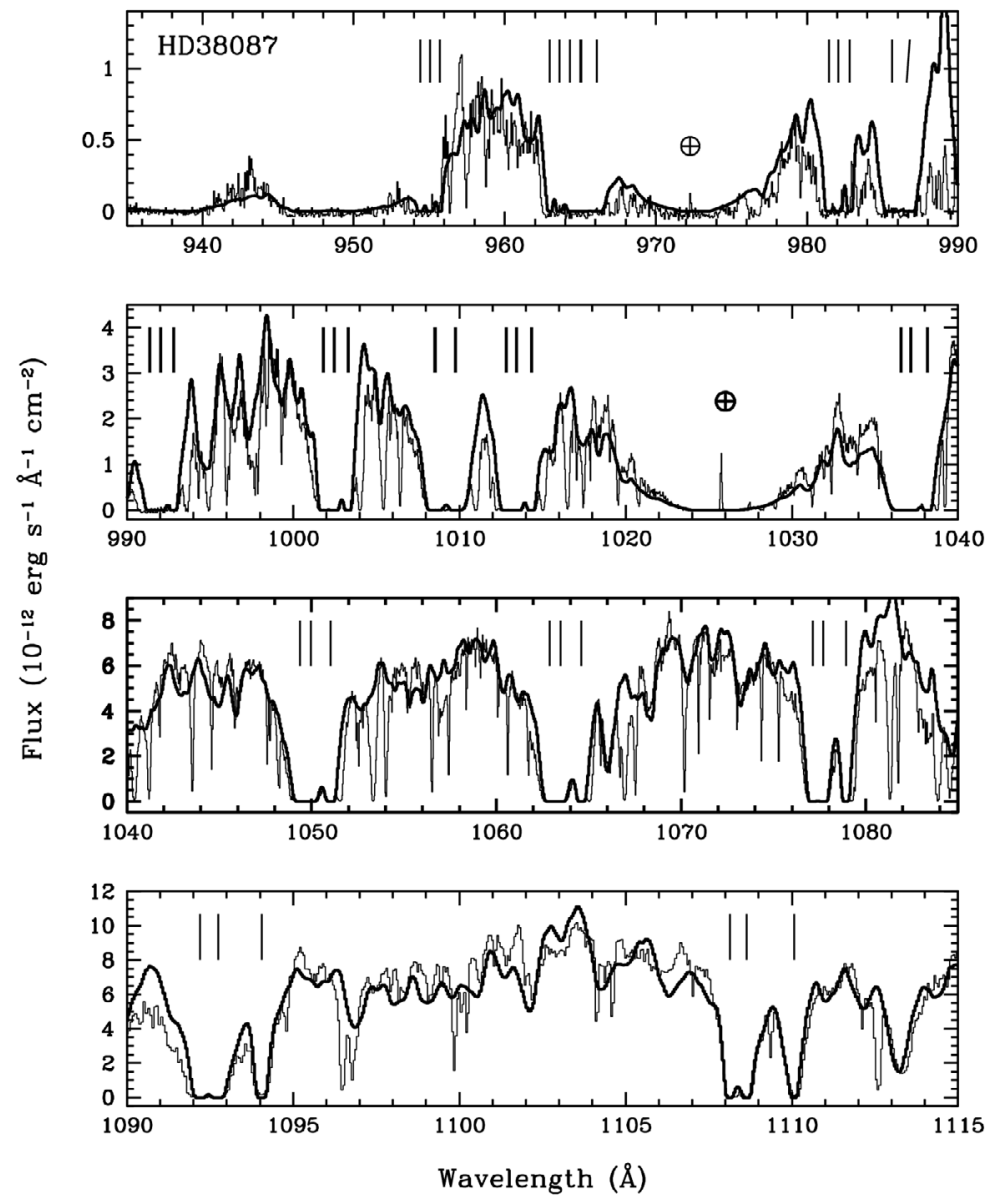

Fig. 12. Overplot of the FUSE spectrum of HD 38087 (thin line) and the synthetic spectrum obtained by combining our photospheric model with the results of the Meudon PDR Code (thick line). Same legend as for Fig. 8. 